\title{
OPEN The structure-based cancer-related single amino acid variation prediction
}

\author{
Jia-Jun Liu ${ }^{1}$, Chin-Sheng Yu ${ }^{2,3}$, Hsiao-Wei Wu ${ }^{4}$, Yu-Jen Chang ${ }^{1}$, Chih-Peng Lin ${ }^{5}$ \& \\ Chih-Hao Lu ${ }^{1,4,6 \bowtie}$
}

Single amino acid variation (SAV) is an amino acid substitution of the protein sequence that can potentially influence the entire protein structure or function, as well as its binding affinity. Protein destabilization is related to diseases, including several cancers, although using traditional experiments to clarify the relationship between SAVs and cancer uses much time and resources. Some SAV prediction methods use computational approaches, with most predicting SAV-induced changes in protein stability. In this investigation, all SAV characteristics generated from protein sequences, structures and the microenvironment were converted into feature vectors and fed into an integrated predicting system using a support vector machine and genetic algorithm. Critical features were used to estimate the relationship between their properties and cancers caused by SAVs. We describe how we developed a prediction system based on protein sequences and structure that is capable of distinguishing if the SAV is related to cancer or not. The five-fold cross-validation performance of our system is $\mathbf{8 9 . 7 3 \%}$ for the accuracy, 0.74 for the Matthews correlation coefficient, and 0.81 for the F1 score. We have built an online prediction server, CanSavPre (http://bioinfo.cmu.edu.tw/CanSavPre/), which is expected to become a useful, practical tool for cancer research and precision medicine.

Single amino acid variation (SAV) refers to one amino acid substitution resulting from genetic polymorphisms. Nonsynonymous encoding variants alter the protein sequence. In extreme cases, this alteration affects the entire protein structure or function. The unique physicochemical properties of each type of amino acid means that the occurrence of the mutation in different positions of the sequence affects protein conformation and its function to different extents. It is vital to understand how the SAV influences protein and to clarify the links between genetic variations and human diseases. Most disease-related SAVs occur in structurally or functionally essential positions $^{1-3}$. Previous research that mapped nonsynonymous SNPs to the structural surfaces of encoded proteins found that about $88 \%$ of disease mutations are located in the voids or pockets ${ }^{4}$. These mutation residues may affect the protein structure or the aggregation of the complex. Importantly, protein destabilization is a primary factor in many Mendelian diseases. Two-thirds of disease-related mutations adversely influence protein-protein interactions via loss of interactions, misfolding, or impaired expression ${ }^{5,6}$.

Structural dynamics are correlated to protein function, with evidence of missense-folding structures resulting in protein dysfunction ${ }^{7,8}$. If missense variants occur at the functional sites, the resulting changes in protein activity and binding affinity cause disease. SAVs located in the protein surfaces are also related to diseases, because these SAVs can destroy protein-protein interactions ${ }^{9,10}$. Increasing evidence indicates that SAVs are associated with several different cancers. Proteins containing harmful amino acid substitutions can affect pathways in different cancers ${ }^{11-13}$. Much evidence has revealed substantial changes in genomic sequences in patients with cancer. Ovarian cancer samples from The Cancer Genome Atlas (TCGA) project, for example, have revealed as many as 4,128 mutations at 575 genes in a cohort of 590 cases $^{14}$. The TCGA project has also identified approximately 9,000 mutations at 575 genes among 564 patients with lung adenocarcinoma ${ }^{15}$. Recent research has suggested that somatic mutation accumulation is critical in tumorigenesis ${ }^{16}$. Moreover, while some variations may appear to be neutral, they may actually be driver mutations that contribute to cancer progression ${ }^{17}$. In the era of precision medicine, it is still difficult to precisely identify which genetic mutation serves as the trigger point of

\footnotetext{
${ }^{1}$ The Ph.D. Program of Biotechnology and Biomedical Industry, China Medical University, Taichung, Taiwan. ${ }^{2}$ Department of Information Engineering and Computer Science, Feng Chia University, Taichung, Taiwan. ${ }^{3}$ Master's Program in Biomedical Informatics and Biomedical Engineering, Feng Chia University, Taichung, Taiwan. ${ }^{4}$ Graduate Institute of Biomedical Sciences, China Medical University, Taichung, Taiwan. ${ }^{5}$ Yourgene Health, New Taipei City, Taiwan. ${ }^{6}$ Department of Medical Laboratory Science and Biotechnology, China Medical University, Taichung, Taiwan. ${ }^{\circledR}$ email: chlu@mail.cmu.edu.tw
} 
tumorigenesis without a systems network biology framework ${ }^{18,19}$. At the proteome level, amino acid substitutions caused by genetic codon transitions may explain the basis of human cancer ${ }^{20}$. Amino acid alterations appear to follow certain rules. For example, arginine has a positive charge that is important for balancing protein and DNA binding; however, arginine is highly mutated in various cancer types. The loss of arginine frequently influences the function of cancer-associated proteins, whereas gaining cysteine, an active and reducing agent, may enhance the capacity of a protein to neutralize reactive oxygen species (ROS) in the tumor environment ${ }^{21-23}$. Proteomic changes caused by proteins carrying missense mutations may help cancer cells adapt to environmental pressure ${ }^{24}$. While different types of cancers have unique properties that are not shared among all cancers, these different cancers may share some substitution patterns ${ }^{25}$. For instance, the amino acid substitution spectrum is similar in breast and digestive tract cancers, and is dominated by the alteration of glutamic acid to lysine ${ }^{25}$. Clarification of the relationship between SAVs and cancers using traditional experiments necessitates much time and many resources, so computational prediction methods are sorely needed in cancer biology research.

Machine learning has become a favored tool for data analysis, as this offers the capacity for leveraging big data and for analyzing the content of complex problems, clarifying information and content ${ }^{26,27}$. Many predictors have emerged that use machine learning as algorithms for SAVs. The two most important and commonly used categories of development strategies are the genetic-based and protein-based prediction systems. Several large-scale sequencing projects such as the TCGA project are widely utilized for genetic analysis ${ }^{28,29}$, some of which follow the American College of Medical Genetics and Genomics (ACMG) guideline ${ }^{30}$. The multifactorial variant prediction (MVP) is a genetic-specific multifactorial model that integrates 16 in silico predictors with the available clinical evidence ${ }^{31}$. However, while the genetic-based system has established a causal relationship between genes and cancer, this relationship is limited to cause and outcome. A comprehensive assessment of cancer driver mutation prediction models evaluated 33 commonly used prediction tools ${ }^{32}$, the top three (CHASM, CTAT-cancer and DEOGEN2) of which are cancer- or protein-based systems ${ }^{33-35}$; CHASM and CTAT-cancer are designed to incorporate the cancer protein, while DEOGEN2 predicts the deleteriousness of SAV by training the mutations in human inherited disease. Since the protein molecule is intimately involved in cellular processes, using the protein-based system to build a prediction system might be more informative for complex diseases such as cancer. However, although numerous prediction models have been developed, we lack the expertise for constructing accurate prediction tools for cancer and knowing which SAV descriptors to incorporate ${ }^{36}$.

We describe our development of a prediction model, which is capable of recognizing whether a particular SAV is cancer-related or is neutral. This model is not only able to discriminate physical changes for each SAV regarding protein function and structure, but it can also estimate how these changes contribute to cancer progression. We hypothesized that accounting for every kind of SAV might be a vital feature for cancer, so we designed a system that incorporates multiple prediction models and enables the user to extract critical features from cancer-related proteins. Our model provides a novel way forward for cancer research, not only for clinical outcomes but also for recognizing prognostic biomarkers, which we contend is a breakthrough for precision medicine.

\section{Materials and methods}

Dataset of SAVs. All SAV data were collected from CanProVar $2.0^{37,38}$, a human Cancer Proteome Variation database that stores both germline and somatic amino acid variations, including those related to the genesis or development of human cancers based on six sources, including the public databases HPI ${ }^{39}, \mathrm{COSMIC}^{40}, \mathrm{OMIM}^{41}$, and TCGA ${ }^{42}$, as well as two large-scale cancer genome resequencing studies ${ }^{43,44}$. CanProVar 2.0 contains 156,671 cancer-related SAVs from mutations that have been reported in cancer samples and 967,017 neutral SAVs from validated coding SNPs in the dbSNP database. In order to determine the exact protein structure of the SAV sequence, CanProVar 2.0 data were mapped to the proteins identified by BLAST ${ }^{45}$ from the Protein Data Bank. The search used six criteria, as follows: 1 . The e-value of alignment results should be smaller than $1 \mathrm{e}-50 ; 2$. The sequence identity of alignment results should be greater than $80 \%$; 3 . The alignment coverage of the protein structure should exceed 95\%; 4. The organism of the aligned target protein should be Homo sapiens; 5 . The experimental method ideally uses X-ray diffraction to extract the protein structure for aligning the target protein structures; and 6. The SAV position should be equidistant between the wild-type in the SAV sequence and the aligned target protein. After matching a protein with a 3-dimensional structure, CD-HIT ${ }^{46}$ was used to filter out homologous proteins. The CD-HIT cluster algorithm generates sets of protein families and uses the sequence identity cut-off of 0.3 to purify redundant proteins. Subsequently, the remaining 2,867 cancer-related SAVs and 7,562 neutral SAVs were our main training set, which were separated into 20 groups by using the representative wild-type amino acid of SAV. The numbers of cancer-related and neutral SAVs for each wild-type amino acid in the training set are listed in Table $1 ; \delta$ is the cancer:neutral SAV ratio, which ranges from 0.2245 to 0.6693 .

Two independent sets were built, independent sets 30 and 40, which collected the proteins filtered out by CDHIT in the previous step. Any proteins in the independent sets sharing more than 30 or $40 \%$ sequence identity with another protein in the same wild-type amino acid group of a training set were filtered out. The final analysis included 154 cancer-related SAVs and 2,240 neutral SAVs in the independent set 30, and 322 cancer-related SAVs and 3,127 neutral SAVs in the independent set 40. Table 1 lists the numbers of cancer-related and neutral SAVs for each wild-type amino acid in the independent sets 30 and 40 . Typically, the $30 \%$ sequence identity was usually used as the criteria to remove homologous proteins. However, due to few cancer-related SAVs were collected in the independent set 30 , we provide the extra independent set 40 for comparison.

Prediction systems. We used the machine learning method to build two cancer-related SAV prediction systems. The first system, CanSavPre ${ }_{\mathrm{w}}$, contained 20 individual prediction models constructed from 20 groups according to the SAV wild-type amino acid. Subsequently, the second prediction system, CanSavPre ${ }_{\mathrm{wm}}$, divided the 20 groups into several subgroups by SAV mutated type. Those subgroups containing fewer than 30 SAVs 


\begin{tabular}{|c|c|c|c|c|c|c|c|c|}
\hline \multirow[b]{2}{*}{ WT } & \multicolumn{4}{|c|}{ Training set } & \multicolumn{2}{|c|}{ Independent set 30} & \multicolumn{2}{|c|}{ Independent set 40} \\
\hline & Cancer & Neutral & Total & Ratio $(\delta)$ & Cancer & Neutral & Cancer & Neutral \\
\hline ALA & 187 & 657 & 844 & 0.2846 & 12 & 191 & 26 & 268 \\
\hline CYS & 45 & 97 & 142 & 0.4639 & 6 & 21 & 9 & 36 \\
\hline ASP & 216 & 412 & 628 & 0.5243 & 14 & 121 & 26 & 167 \\
\hline GLU & 254 & 408 & 662 & 0.6225 & 11 & 114 & 27 & 174 \\
\hline PHE & 85 & 127 & 212 & 0.6693 & 9 & 46 & 14 & 58 \\
\hline GLY & 236 & 437 & 673 & 0.5400 & 11 & 129 & 21 & 171 \\
\hline HIS & 77 & 188 & 265 & 0.4096 & 6 & 63 & 9 & 85 \\
\hline ILE & 116 & 437 & 553 & 0.2654 & 5 & 113 & 9 & 178 \\
\hline LYS & 98 & 263 & 361 & 0.3726 & 6 & 98 & 12 & 131 \\
\hline LEU & 178 & 321 & 499 & 0.5545 & 6 & 99 & 17 & 141 \\
\hline MET & 63 & 202 & 265 & 0.3119 & 2 & 56 & 12 & 83 \\
\hline ASN & 90 & 340 & 430 & 0.2647 & 5 & 107 & 8 & 153 \\
\hline PRO & 200 & 363 & 563 & 0.5510 & 9 & 100 & 24 & 134 \\
\hline GLN & 83 & 212 & 295 & 0.3915 & 7 & 55 & 14 & 78 \\
\hline ARG & 328 & 1,248 & 1,576 & 0.2628 & 14 & 335 & 30 & 459 \\
\hline SER & 217 & 432 & 649 & 0.5023 & 12 & 131 & 25 & 176 \\
\hline THR & 150 & 505 & 655 & 0.2970 & 7 & 146 & 13 & 203 \\
\hline VAL & 156 & 695 & 851 & 0.2245 & 7 & 236 & 16 & 315 \\
\hline TRP & 24 & 42 & 66 & 0.5714 & 1 & 14 & 3 & 22 \\
\hline TYR & 64 & 176 & 240 & 0.3636 & 4 & 65 & 7 & 95 \\
\hline TOTAL & 2,867 & 7,562 & 10,429 & 0.3791 & 154 & 2,240 & 322 & 3,127 \\
\hline
\end{tabular}

Table 1. The numbers of cancer-related and neutral SAVs for each wild-type (WT) residue and the total (bolded) in our training and independent datasets.

were combined with other subgroups that had substitution scores exceeding zero based on BLOSUM62. Any subgroups that did not fit these criteria were omitted from the CanSavPre ${ }_{\mathrm{wm}}$, construction and were instead included in the CanSavPre ${ }_{\mathrm{w}}$ prediction models. The dataset was split into subgroups to determine the characteristics of specific wild-type amino acid alterations in each of the sequence-based, structure-based and microenvironment-based feature sets. As an illustration, a glycine would be built into prediction models containing for example acidic (e.g., aspartic or glutamic acid) and basic (e.g., arginine) mutated amino acids with distinct SAV features. Thus, our feature selection process effectively detects essential features. The second prediction system ultimately yielded 100 prediction models (see Supplementary Table S1).

Each prediction model was a two-level Support Vector Machine (SVM) classifier module. The SVM machine learning method is widely used for classifying protein structure or function in computational biology $\mathrm{y}^{47-52}$. All SVM calculations were performed using LIBSVM (version 3.24 $)^{53,54}$, with the radial basis function (RBF) kernel. The first-level SVM comprised 12 SVM classifiers based on four repeats for three feature sets, which are described in the next section. All SAV descriptors in each feature set were fed into the SVM, and a five-fold cross-validation was performed during model training. The parameters (penalty and gamma values of the RBF kernel) were both trained by exponentially increasing the grid search from $2^{-15}$ to $2^{15}$ incorporating best values of informative measures.

The genetic algorithm (GA) $)^{55,56}$ was used to select features and optimize performance. The basic GA procedures are as follows: $N$ solutions $\left(S_{i}, i=1, \ldots, N\right)$ are randomly generated as the starting population. Each solution $S_{i}$ is represented as a set of vectors $S_{i}=\left(\Phi^{i}\right)$. The feature vector $\Phi^{i}$ is an $m$-dimensional vector, indicating the binary representations of $m$ features: If $f_{j}^{i}=1$, the $j$ th feature is kept; if $f_{j}^{i}=0$, the feature $j^{t h}$ is eliminated. In order to avoid any imbalance between positives and negatives in performance, four informative measures (Eqs. 1-4) for prediction performance were used as the fitness functions, consisting of accuracy (Acc), the Matthews correlation coefficient (MCC), the F1 score (F1), and summation of sensitivity and weighted specificity (Hybrid). They were calculated as follows:

$$
\begin{gathered}
A c c=\frac{T P+T N}{T P+T N+F P+F N}, \\
M C C=\frac{T P \times T N-F P \times F N}{\sqrt{(T P+F P)(T P+F N)(T N+F P)(T N+F N)}}, \\
F 1=\frac{2 \times \text { Precision } \times \text { Sensitivity }}{\text { Precision }+ \text { Sensitivity }},
\end{gathered}
$$




$$
\text { Hybrid }=\text { Sensitivity }+\delta \times \text { Specificity, }
$$

where Precision $=\frac{T P}{T P+F P}$, Sensitivity $=\frac{T P}{T P+F N}$, Specificity $=\frac{T N}{T N+F P}, T P$ represents true-positives, $T N$ represents true-negatives, $F P$ represents false-positives, $F N$ represents false-negatives and $\delta$ is the ratio of the number of cancer-related SAVs to neutral SAVs, which are listed in Table 1.

In the initial population, $N$ solutions are randomly divided into two halves. $\alpha$ and $\beta$ have the best fitness in each half, and they are defined as $\alpha=\left(\Phi^{\alpha}\right)=\max \left\{S_{1}, \ldots, S_{N / 2}\right\}$ and $\beta=\left(\Phi^{\beta}\right)=\max \left\{S_{\frac{N}{2}+1}, \ldots, S_{N}\right\}$. In general, the three basic mechanisms driving the evolutionary processes in one generation consist of the selection, mutation and crossover processes.

Selection operator. In the $\tau^{\text {th }}$ generation, the selection operators are defined as:

$$
\begin{aligned}
\alpha^{\tau} & =\max \left\{S_{1}^{\tau-1}, \ldots, S_{N / 2}^{\tau-1}, \alpha^{\tau-1}\right\}, \\
\beta^{\tau} & =\max \left\{S_{\frac{N}{2}+1}^{\tau-1}, \ldots, S_{N}^{\tau-1}, \beta^{\tau-1}\right\} .
\end{aligned}
$$

Note that for the special case of $\tau=0, \alpha^{0}$ and $\beta^{0}$ are defined as 0 . A new solution, $S_{i}^{\tau}$, is equal to $\alpha^{\tau}$ if $i$ is odd, while $S_{i}^{\tau}$ is equal to $\beta^{\tau}$ if $i$ is even.

Mutation operator. We apply two types of mutation to the $N$ solutions $S_{i}$ s. In the case of $i=1, \ldots, N / 2$, every $b$ bit of the vectors is subject to mutation: $b=\sim b$, if the mutation rate is less than a mutation threshold $\mu_{0}=0.1$. In the case of $i=\frac{N}{2}+1, \ldots, N$, we randomly choose a bit from the vectors. These bits are then subject to mutation without any mutation thresholds.

Crossover operators. The crossover operations are carried out between $S_{2 p-1}$ and $S_{2 p}$, where $p=1, \ldots, N / 2$ and proceed as follows: one-point crossover is performed between $\Phi^{2 p-1}$ and $\Phi^{2 p}$ if the crossover rate is less than the crossover threshold $\mu_{1}=0.5$.

The second level of SVM classifiers is used to process the prediction results generated from 12 classifiers in the first level, to produce the final probability distribution of the relationship with cancer-related SAVs or neutral SAVs. The relationship with the largest probability is used as the final prediction. The two-level SVM system is shown schematically in Fig. 1.

Classification of feature sets. SAV descriptors for machine learning were classified into three classes: sequence-based, structure-based, and microenvironment-based feature sets. For the sequence-based feature set, 44 descriptors were extracted from the protein sequence and approximately partitioned into three categories. The first category contained the most commonly used substitution index of wild-type SAV residues to mutations. Three kinds of substitution indices were used; the BLOSUM62 ${ }^{57,58}$, PAM250 ${ }^{59}$, and the position-specific scoring matrix (PSSM), which was derived from PSI-BLAST ${ }^{60}$. The second category represented the conservation for each residue compared with homologs. The 15 evolutional entropy values derived from PSI-BLAST were used to denote a sliding window containing 7 amino acids on either side of the SAV. The model also calculated average entropy values for window lengths 5 and 15 , centered on the SAV, representing local- and wide-ranging sequence conservation, respectively. In the third category, an amino acid composition (AAC) ${ }^{61}$, a 15-residue peptide (with 7 amino acids on either side of the SAV), represented the composition of the neighboring residues. According to the physicochemical properties of residues, we used the following classification schemes ${ }^{62}$ of amino acid compositions: $\mathbf{H}$ for polar (RKEDQN), neutral (GASTPHY), and hydrophobic (CVLIMFW); $\mathbf{V}$ for small (GASCTPD), medium (NVEQIL), and large (MHKFRYW); $\mathbf{Z}$ for low (GASDT), medium (CPNVEQIL), and high polarizability (KMHFRYW); P for low (LIFWCMVY), neutral (PATGS), and high polarity (HQRKNED); F for acidic (DE), basic (HKR), polar (CGNQSTY), and nonpolar (AFILMPVW); E for acidic (DE), basic (HKR), aromatic (FWY), amide (NQ), small hydroxyl (ST), sulfur-containing (CM), aliphatic 1 (AGP), and aliphatic 2 (ILV). For clarity, these sequence-based descriptors are summarized in Table 2.

The structure-based feature sets contained 13 descriptors extracted from PDB and DSSP ${ }^{63,64}$. The first structure-based descriptor used the B-factor value of the SAV C $\alpha$ atom; the B-factor value represents those atoms displaced from their mean positions in a crystal structure diminishes the scattered X-ray intensity. This displacement may be the result of temperature-dependent atomic vibrations, or static disorder in a crystal lattice. Our model also uses critical DSSP information regarding solvent accessibility and includes eight DSSP-defined elements in the secondary structure (i.e., H, B, E, G, I, T, S, and others), energy from the acceptor and donor backbone hydrogen bonds, and determines whether or not disulfide bonding exists. These structure-based descriptors are summarized in Table 3.

In the third feature set, the weighted contact number (WCN) model ${ }^{65}$ was used to describe the microenvironment properties of SAVs. This weighted contact number model has a local packing density profile, and research has reported a high correlation between the WCN profile and the sequence conservation profile ${ }^{66}$. The WCN value of atom $i$ was calculated by $W_{C N}=\sum_{j \neq i}^{N} 1 / r_{i j}^{2}$, where $r_{i j}$ was the distance between the atom $i$ and atom $j$, while $N$ was the number of calculated atoms. In this work, atom $i$ was defined as the C $\alpha$ atom of SAV, and the different microenvironment properties were represented by calculated different atom types, or the source of atom 


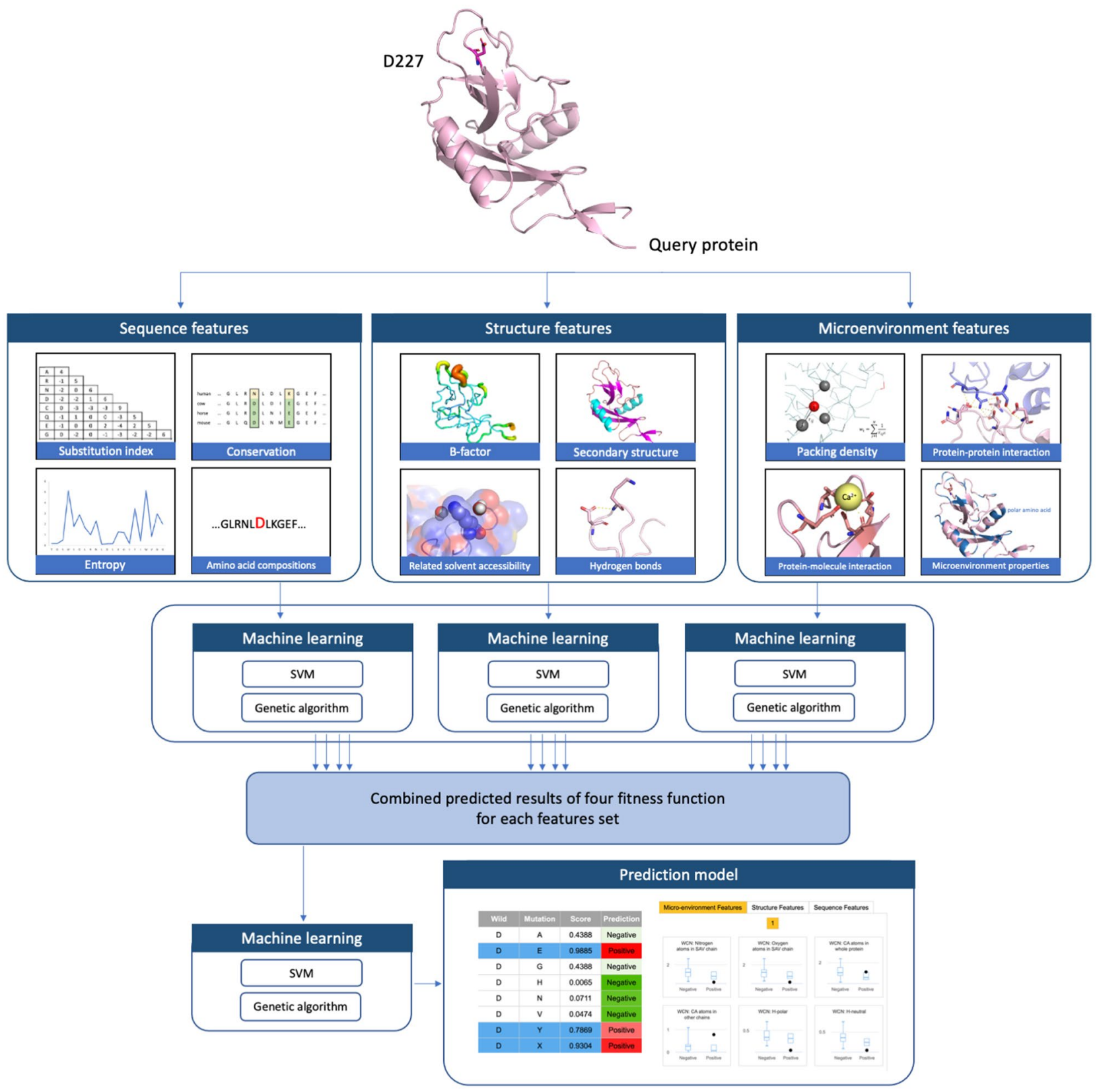

Figure 1. The workflow diagram represents the two-level SVM prediction system.

$j$. The atom type of $j$ could be $\mathrm{C} \alpha$ atoms, nitrogen atoms or oxygen atoms of an amino acid. Moreover, the source of atom $j$ could be located within the same protein chain as SAV or the whole protein, representative of SAV packing density. Alternatively, the source could be derived from another protein chain or molecules such as DNA, RNA, ligands, or metal ions representing protein-protein or protein-molecule interactions. The packing density of SAV may be divided into different classifications representing the microenvironment properties wherein the SAV is located, such as polar, hydrophobic, acidic or basic, according to the physicochemical properties of residues containing the $\mathrm{C} \alpha$ atom $j$. The same classification schemes were used as described in the sequencebased feature set. The microenvironment-based descriptors are listed in Table 4.

\section{Results}

Performance evaluation. Table 5 compares the five-fold cross-validation performances of two prediction systems based on three different feature sets with the prediction performance of the second-level SVM, all of which are optimized by using MCC as the fitness function in GA. In our experiment, the individual prediction model using the sequence-based feature scheme outperformed the other two, while the performance of the model using the microenvironment-based feature was superior to that of the structure-based feature scheme. The outstanding performance of the combined model obtained in the second-level SVM procedure demonstrates that further information is very helpful for understanding and determining cancer-related factors. 


\begin{tabular}{|l|l|l|l|}
\hline$\#$ & Feature name & $\#$ & Feature name \\
\hline 1 & Substitution index: BLOSUM62 & 23 & AAC: $H$-hydrophobic (CVLIMFW) \\
\hline 2 & Substitution index: PAM250 & 24 & AAC: $V$-small (GASCTPD) \\
\hline 3 & Substitution index: PSSM & 25 & AAC: $V$-medium (NVEQIL) \\
\hline 4 & Entropy: 7th residue before SAV & 26 & AAC: $V$-large (MHKFRYW) \\
\hline 5 & Entropy: 6th residue before SAV & 27 & AAC: $Z$-low polarizability (GASDT) \\
\hline 6 & Entropy: 5th residue before SAV & 28 & AAC: $Z$-neutral (PATGS) \\
\hline 7 & Entropy: 4 th residue before SAV & 29 & AAC: $Z$-high polarizability (KMHFRYW) \\
\hline 8 & Entropy: 3rd residue before SAV & 30 & AAC: $P$-low polarity (LIFWCMVY) \\
\hline 9 & Entropy: 2 nd residue before SAV & 31 & AAC: $P$-neutral polarity (PATGS) \\
\hline 10 & Entropy: 1 st residue before SAV & 32 & AAC: $P$-high polarity (HQRKNED) \\
\hline 11 & Entropy: SAV & 33 & AAC: $F$-acidic (DE) \\
\hline 12 & Entropy: 1 st residue after SAV & 34 & AAC: $F$-basic (HKR) \\
\hline 13 & Entropy: 2 nd residue after SAV & 35 & AAC: $F$-polar (CGNQSTY) \\
\hline 14 & Entropy: 3 rd residue after SAV & 36 & AAC: $F$-nonpolar (AFILMPVW) \\
\hline 15 & Entropy: 4th residue after SAV & 37 & AAC: $E$-acidic (DE) \\
\hline 16 & Entropy: th residue after SAV & 38 & AAC: $E$-basic (HKR) \\
\hline 17 & Entropy: 6th residue after SAV & 39 & AAC: $E$-aromatic (FWY) \\
\hline 18 & Entropy: 7th residue after SAV & 40 & AAC: $E$-amide (NQ) \\
\hline 19 & Entropy: Average of 15 residues & 41 & AAC: $E$-small hydroxyl (ST) \\
\hline 20 & Entropy: Average of 5 residues & 42 & AAC: $E$-sulfur-containing (CM) \\
\hline 21 & AAC: $H$-polar (RKEDQN) & 43 & AAC: $E$-aliphatic 1 (AGP) \\
\hline 22 & AAC: $H$-neutral (GASTPHY) & 44 & AAC: $E$-aliphatic 2 (ILV) \\
\hline & & & \\
\hline
\end{tabular}

Table 2. List of descriptors in the sequence-based feature set.

\begin{tabular}{|l|l|l|l|}
\hline$\#$ & Feature name & $\#$ & Feature name \\
\hline 1 & B-factor & 8 & Secondary structure: T \\
\hline 2 & Related solvent accessibility & 9 & Secondary structure: S \\
\hline 3 & Secondary structure: H & 10 & Secondary structure: Others \\
\hline 4 & Secondary structure: B & 11 & Energy of backbone H-bond: acceptor \\
\hline 5 & Secondary structure: E & 12 & Energy of Backbone H-bond: donor \\
\hline 6 & Secondary structure: G & 13 & Disulfide bond \\
\hline 7 & Secondary structure: I & & \\
\hline
\end{tabular}

Table 3. List of descriptors in the structure-based feature set.

Critically, CanSavPre ${ }_{\mathrm{wm}}$ performed better than CanSavPre ${ }_{\mathrm{w}}$ in all three individual feature sets and also in the combined set. Specific training and predicting models were built from the specific subgroups according to the wild-type and mutated types of SAV. Using a two-level SVM combining sequence-, structure- and microenvironment-based features, CanSavPre ${ }_{\mathrm{w}}$ distinguished between SAVs that were or were not related to cancer, with an accuracy of $79.83 \%$, a Matthews correlation coefficient of 0.45 , and F1 score of 0.54 . CanSavPre wm $_{\text {is more }}$ effective, with an accuracy of $89.73 \%$, a Matthews correlation coefficient of 0.74 , and F1 score of 0.81 . The fivefold

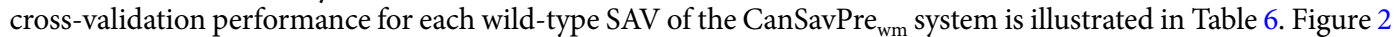
illustrates the ROC curve and compares the AUC values of each wild-type SAV in two systems.

Case study: PI3K. The phosphatidylinositol-3-kinase (PI3K) signaling pathway contributes to several cellular processes, including metabolism, proliferation, differentiation and activation. Notably, the PI3K/AKT/mammalian target of rapamycin (mTOR) signaling pathway is one of the most important intracellular pathways and is also one of the most frequently dysregulated pathways in human cancers ${ }^{67-70}$. Several catalysis subunits exist for PI3K. Those that are encoded by PIK $3 \mathrm{c} \delta$ have been found to induce cell proliferation in colorectal cancer and other types of cancers ${ }^{71,72}$. The amino acid mutation of $\mathrm{PI} 3 \mathrm{Kc} \delta$ is closely related to oncogenic transformation, and numerous SAVs have been recorded as cancer-related in the COSMIC database, including P57S, Q75K, K111E, P134L, S361F, N380H, L634F, H677R, E713K, A723V, I776T, G890R, and L977I. Figure 3 illustrates the protein structures of PI3K and the p $85 \alpha$ complex (PDB ID: 5DXU) 73 ; 14 amino acids, including one neutral and 13 cancer-related SAVs, are drawn as spheres. All SAVs, except H677R, are correctly predicted by our prediction system. It should be noted that another SAV, R104C, has been marked as a neutral SAV and is also predicted correctly. The predicted results of PI3K are listed in Table 7. 


\begin{tabular}{|l|l|l|l|}
\hline$\#$ & Feature name & $\#$ & Feature name \\
\hline 1 & WCN: $C_{\alpha}$ atoms in SAV chain & 17 & WCN: $Z$-high polarizability (KMHFRYW) \\
\hline 2 & WCN: Nitrogen atoms in SAV chain & 18 & WCN: $P$-low polarity (LIFWCMVY) \\
\hline 3 & WCN: Oxygen atoms in SAV chain & 19 & WCN: $P$-neutral polarity (PATGS) \\
\hline 4 & WCN: $C_{\alpha}$ atoms in whole protein & 20 & WCN: $P$-high polarity (HQRKNED) \\
\hline 5 & WCN: Nitrogen in whole protein & 21 & WCN: $F$-acidic (DE) \\
\hline 6 & WCN: Oxygens in whole protein & 22 & WCN: $F$-basic (HKR) \\
\hline 7 & WCN: $C_{\alpha}$ atoms in other chains & 23 & WCN: $F$-polar (CGNQSTY) \\
\hline 8 & WCN: Atoms in other molecules & 24 & WCN: $F$-nonpolar (AFILMPVW) \\
\hline 9 & WCN: $H$-polar (RKEDQN) & 25 & WCN: $E$-acidic (DE) \\
\hline 10 & WCN: $H$-neutral (GASTPHY) & 26 & WCN: $E$-basic (HKR) \\
\hline 11 & WCN: $H$-hydrophobic (CVLIMFW) & 27 & WCN: $E$-aromatic (FWY) \\
\hline 12 & WCN: $V$-small (GASCTPD) & 28 & WCN: $E$-amide (NQ) \\
\hline 13 & WCN: $V$-medium (NVEQIL) & 29 & WCN: $E$-small hydroxyl (ST) \\
\hline 14 & WCN: $V$-large (MHKFRYW) & 30 & WCN: $E$-sulfur-containing (CM) \\
\hline 15 & WCN: $Z$-low polarizability (GASDT) & 31 & WCN: $E$-aliphatic 1 (AGP) \\
\hline 16 & WCN: $Z$-neutral (PATGS) & 32 & WCN: $E$-aliphatic 2 (ILV) \\
\hline
\end{tabular}

Table 4. List of descriptors in the microenvironment-based feature set.

\begin{tabular}{|l|l|l|l|l|l|l|l|}
\hline System & Feature set & Accuracy & Sensitivity & Specificity & MCC & Precision & F1 score \\
\hline \multirow{4}{*}{ CanSavPre $_{\mathrm{w}}$} & Sequence & 0.7450 & 0.4350 & 0.8620 & 0.3202 & 0.5434 & 0.4832 \\
\cline { 2 - 8 } & Structure & 0.6995 & 0.3946 & 0.8146 & 0.2176 & 0.4454 & 0.4185 \\
\cline { 2 - 8 } & Microenvironment & 0.7184 & 0.3988 & 0.8391 & 0.2536 & 0.4832 & 0.4370 \\
\cline { 2 - 8 } & Combined & 0.7983 & 0.4356 & 0.9357 & 0.4452 & 0.7199 & 0.5428 \\
\hline \multirow{3}{*}{ CanSavPre $_{\text {wm }}$} & Sequence & 0.8471 & 0.6292 & 0.9293 & 0.5978 & 0.7706 & 0.6928 \\
\cline { 2 - 8 } & Structure & 0.8022 & 0.4737 & 0.9262 & 0.4609 & 0.7078 & 0.5676 \\
\cline { 2 - 8 } & Microenvironment & 0.8311 & 0.5774 & 0.9268 & 0.5509 & 0.7487 & 0.6520 \\
\cline { 2 - 8 } & Combined & 0.8973 & 0.7837 & 0.9404 & 0.7382 & 0.8328 & 0.8075 \\
\hline
\end{tabular}

Table 5. Comparisons of the five-fold cross-validation performance values in the training set of two prediction systems based on three feature sets, combined by second-layer SVM. All predictions were optimized using MCC as the fitness function.

Case study: D227Y of CD23. CD23 is the low-affinity receptor for IgE and is expressed on the surface of several hematopoietic cells ${ }^{74}$, such as lymphocytes ${ }^{75}$, monocytes ${ }^{76}$, follicular dendritic cells ${ }^{77,78}$, and bone marrow stromal cells ${ }^{79}$. Several stimuli regulate the expression of CD23, a critical factor for B-cell activation, growth, and IgE production (OMIM\#151445). The D227Y mutation arising from an alteration of the FCER2 gene has been reported in head and neck squamous cell carcinoma (HNSCC) $)^{80}$ and the colorectal neuroendocrine carcinoma mutational analyses project ${ }^{81}$. D227 is located in one of the conserved double-loops; the interface between CD23 and the carbohydrate protein, Fce 3-4. Importantly, calcium $\left(\mathrm{Ca}^{2+}\right)$ is a regulated ligand for CD23 binding affinity and $\mathrm{Ca}^{2+}$ binding enables loop1 and loop4 to change the conformation and increase the binding affinity. D227 (loop1) and D258 (loop4) form additional salt bridges between CD23 and Fc $3-4^{82,83}$. Other bounds are involved in CD23 and Fce 3-4 binding, while D227Y affects the binding affinity and IgE antitumor functioning (Fig. 4).

The boxplots of microenvironment descriptors in Fig. 4 depict the subgroup with the ASP to the TYR mutation. In several descriptors in Fig. 5, the distribution of cancer-related SAVs differs significantly from that of the neutral SAVs, with a $95 \%$ confidence interval by two sample $z$-test $(z$ score $>1.96, p<0.05)$. The $z$ score is defined by $z=\frac{\left(x_{1}-x_{2}\right)-\left(\mu_{1}-\mu_{2}\right)}{\sqrt{\frac{\sigma_{1}^{2}}{n_{1}}-\frac{\sigma_{2}^{2}}{n_{2}}}}$, where $\left(x_{1}-x_{2}\right)$ and $\left(\mu_{1}-\mu_{2}\right)$ are the observed and expected differences between cancerrelated and neutral SAVs, respectively. $\sigma_{1}$ and $n_{1}$ are the standard error and amount for the cancer-related SAVs group, and $\sigma_{2}$ and $n_{2}$ are for the neutral SAVs group. The cancer-related SAVs are located in the relatively low packing density region, encompassing $\mathrm{C} \alpha$ atoms, nitrogen, or oxygen located in a single SAV chain, or in whole protein. D227Y in CD23 has a low WCN value in a single SAV chain but a relatively high WCN value in whole protein or other chains (Fig. 5a-c), because D227 is located in the interface of CD23 and FcE 3-4, and is involved in their binding. Subsequently, the cancer-related SAVs have lower distributions of H-neutral (AGPHY), V-small (GASCTPD), V-large (MHKFRYW), Z-low polarizability (GASDT), Z-high polarizability (KMHFRYW), P-neutral polarity (PATGS), F-basic (HKR), F-nonpolar (AFILMPVW), E-basic (HKR), E-aromatic (FWY) and E-aliphatic1 (AGP) with the surrounding amino acids. This unique surrounding pattern is also found in the case of D227Y in CD23 (Fig. 5d-i). 


\begin{tabular}{|l|l|l|l|l|l|l|l|l|l|l|}
\hline WT & TP & TN & FP & FN & Accuracy & Sensitivity & Specificity & MCC & Precision & F1 score \\
\hline ALA & 119 & 616 & 41 & 68 & 0.8709 & 0.6364 & 0.9376 & 0.6081 & 0.7438 & 0.6859 \\
\hline CYS & 43 & 93 & 4 & 2 & 0.9577 & 0.9556 & 0.9588 & 0.9040 & 0.9149 & 0.9348 \\
\hline ASP & 160 & 375 & 37 & 56 & 0.8519 & 0.7407 & 0.9102 & 0.6664 & 0.8122 & 0.7748 \\
\hline GLU & 212 & 341 & 67 & 42 & 0.8353 & 0.8346 & 0.8358 & 0.6602 & 0.7599 & 0.7955 \\
\hline PHE & 67 & 118 & 9 & 18 & 0.8726 & 0.7882 & 0.9291 & 0.7331 & 0.8816 & 0.8323 \\
\hline GLY & 192 & 406 & 31 & 44 & 0.8886 & 0.8136 & 0.9291 & 0.7528 & 0.8610 & 0.8366 \\
\hline HIS & 69 & 182 & 6 & 8 & 0.9472 & 0.8961 & 0.9681 & 0.8710 & 0.9200 & 0.9079 \\
\hline ILE & 97 & 428 & 9 & 19 & 0.9494 & 0.8362 & 0.9794 & 0.8436 & 0.9151 & 0.8739 \\
\hline LYS & 80 & 258 & 5 & 18 & 0.9363 & 0.8163 & 0.9810 & 0.8357 & 0.9412 & 0.8743 \\
\hline LEU & 154 & 292 & 29 & 24 & 0.8938 & 0.8652 & 0.9097 & 0.7702 & 0.8415 & 0.8532 \\
\hline MET & 55 & 199 & 3 & 8 & 0.9585 & 0.8730 & 0.9851 & 0.8835 & 0.9483 & 0.9091 \\
\hline ASN & 78 & 333 & 7 & 12 & 0.9558 & 0.8667 & 0.9794 & 0.8643 & 0.9176 & 0.8914 \\
\hline PRO & 150 & 324 & 39 & 50 & 0.8419 & 0.7500 & 0.8926 & 0.6512 & 0.7937 & 0.7712 \\
\hline GLN & 68 & 200 & 12 & 15 & 0.9085 & 0.8193 & 0.9434 & 0.7714 & 0.8500 & 0.8344 \\
\hline ARG & 216 & 1,189 & 59 & 112 & 0.8915 & 0.6585 & 0.9527 & 0.6538 & 0.7855 & 0.7164 \\
\hline SER & 202 & 376 & 56 & 15 & 0.8906 & 0.9309 & 0.8704 & 0.7724 & 0.7829 & 0.8505 \\
\hline THR & 118 & 490 & 15 & 32 & 0.9282 & 0.7867 & 0.9703 & 0.7907 & 0.8872 & 0.8339 \\
\hline VAL & 97 & 681 & 14 & 59 & 0.9142 & 0.6218 & 0.9799 & 0.6912 & 0.8739 & 0.7266 \\
\hline TRP & 23 & 41 & 1 & 1 & 0.9697 & 0.9583 & 0.9762 & 0.9345 & 0.9583 & 0.9583 \\
\hline TYR & 47 & 169 & 7 & 17 & 0.9000 & 0.7344 & 0.9602 & 0.7356 & 0.8704 & 0.7966 \\
\hline TOTAL & $\mathbf{2 , 2 4 7}$ & $\mathbf{7 , 1 1 1}$ & $\mathbf{4 5 1}$ & $\mathbf{6 2 0}$ & $\mathbf{0 . 8 9 7 3}$ & $\mathbf{0 . 7 8 3 7}$ & $\mathbf{0 . 9 4 0 4}$ & $\mathbf{0 . 7 3 8 2}$ & $\mathbf{0 . 8 3 2 8}$ & $\mathbf{0 . 8 0 7 5}$ \\
\hline
\end{tabular}

Table 6. The five-fold cross-validation performance values in the training set of the CanSavPre ${ }_{\mathrm{wm}}$ system for each wild-type (WT) residue and the total (bolded).
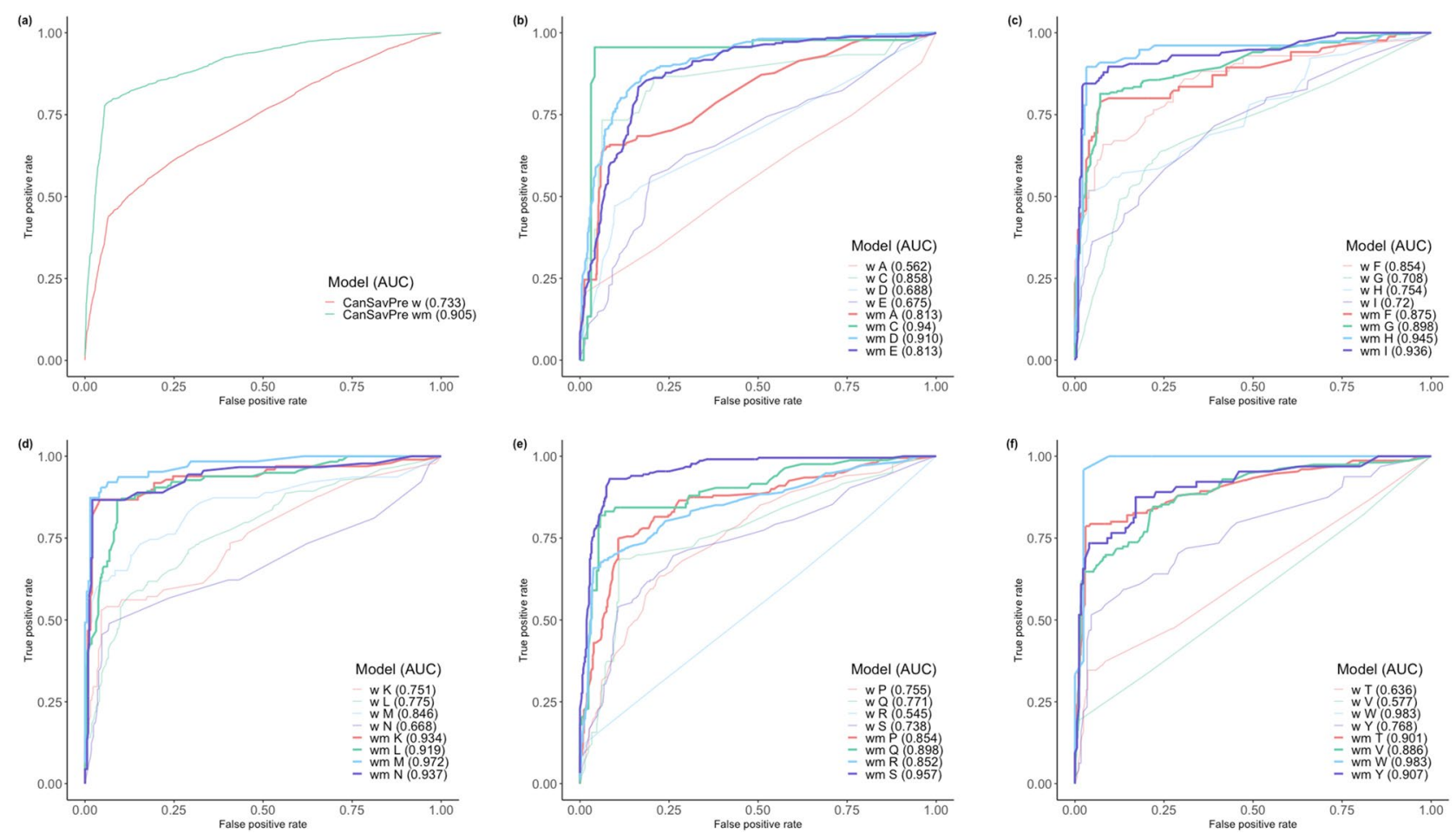

Figure 2. Training set ROC curves in two prediction systems. (a) Total groups in the two systems; (b) A, C, D, E groups; (c) F, G, H, I groups; (d) K, L, M, N groups; (e) P, Q, R, S groups; (f) T, V, W, Y groups. 


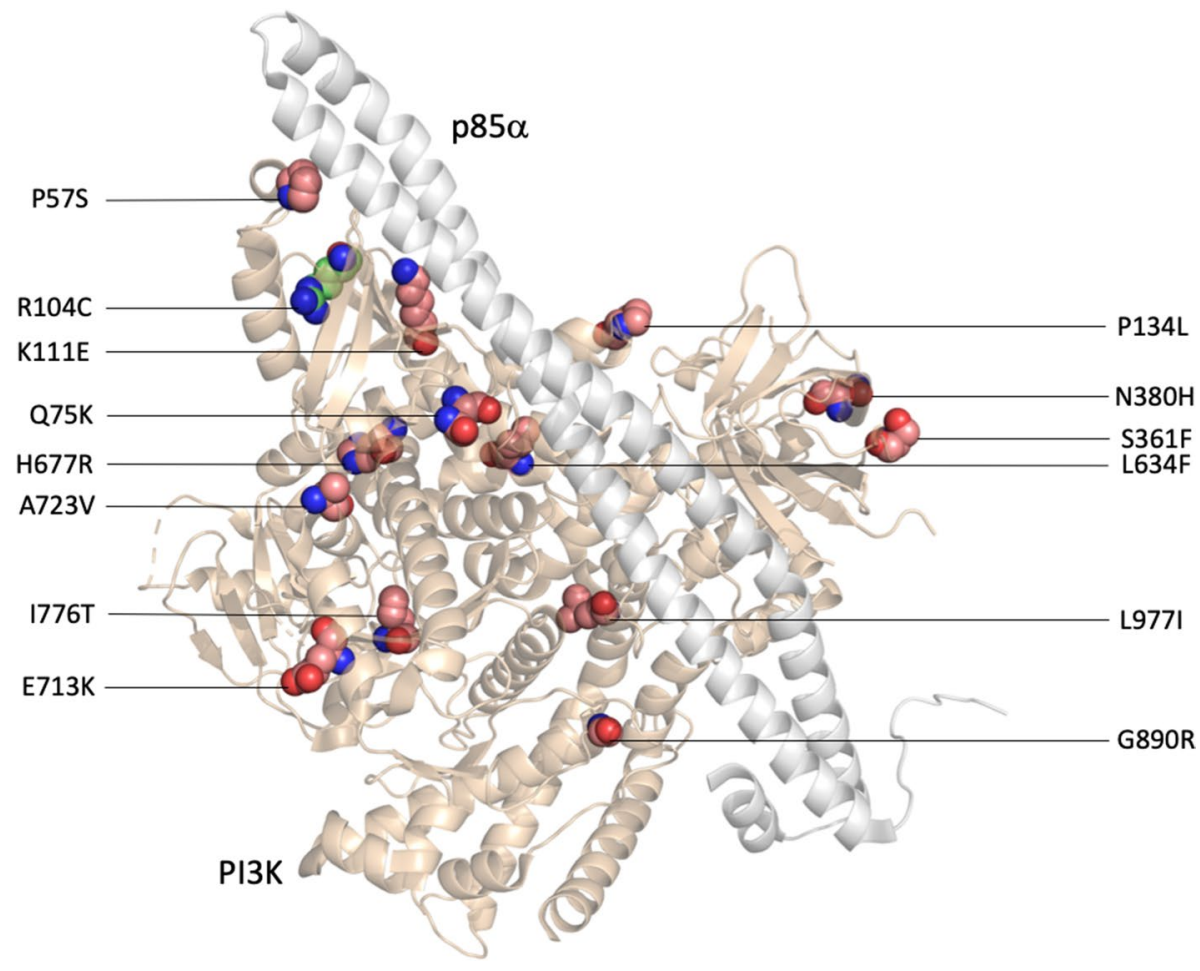

Figure 3. Protein structures of the PI3K/p $85 \alpha$ complex. The PI3K (wheat color) and p $85 \alpha$ (gray color) complex (PDB ID: 5DXU) in the cartoon is drawn by PyMOL ${ }^{100}$. ARG104 (illustrated by the green spheres) is a neutral SAV that mutates to CYS. The other residues shown in the pink spheres are all cancer-related SAVs.

\begin{tabular}{|l|l|l|l|}
\hline SAV & Type & Score & Predicted results \\
\hline P57S & Cancer-related & 0.8590 & TP \\
\hline Q75K & Cancer-related & 0.9973 & TP \\
\hline R104C & Neutral & 0.1016 & TN \\
\hline K111E & Cancer-related & 0.6590 & TP \\
\hline P134L & Cancer-related & 0.7545 & TP \\
\hline S361F & Cancer-related & 0.9408 & TP \\
\hline N380H & Cancer-related & 0.9521 & TP \\
\hline L634F & Cancer-related & 0.8879 & TP \\
\hline H677R & Cancer-related & 0.0507 & FN \\
\hline E713K & Cancer-related & 0.8438 & TP \\
\hline A723V & Cancer-related & 0.8595 & TP \\
\hline I776T & Cancer-related & 0.9656 & TP \\
\hline G890R & Cancer-related & 0.9350 & TP \\
\hline L977I & Cancer-related & 0.8000 & TP \\
\hline
\end{tabular}

Table 7. Predicted results for the 14 SAVs of the PI3K.

Case study: E194G of CASQ. The calsequestrin (CASQ) is a $\mathrm{Ca}^{2+}$ buffering protein, capable of storing large amounts of $\mathrm{Ca}^{2+}$ in cardiac and skeletal muscles. $\mathrm{Ca}^{2+}$ is an essential molecule that can regulate diverse cellular processes, such as gene transcription, cell proliferation and migration ${ }^{84-86}$. Although most research into the CASQ has focused on cardiac muscle, CASQ in the $\mathrm{Ca}^{2+}$ signaling pathway is also vital in cancer research ${ }^{87}$, as this pathway is highly correlated with tumor growth and metastasis ${ }^{88}$. Importantly, T189, E194 and D196 can form a pack in the CASQ that harbors $\mathrm{Ca}^{2+89}$; this $\mathrm{Ca}^{2+}$ binding can be destroyed by the substitution E194G, causing the protein to lose its functionality (Fig. 6).

In the subgroup in which GLU mutates to GLY, none of the microenvironment descriptors of cancer-related or neutral SAVs reveal significant differences at the $95 \%$ confidence interval, although several relevant descriptors are found in the case of E194G in the CASQ. That is, E194 exhibits higher WCN values of oxygen in a single SAV chain, as well as higher WCN values of atoms in other molecules, due to the fact that the CASQ is 


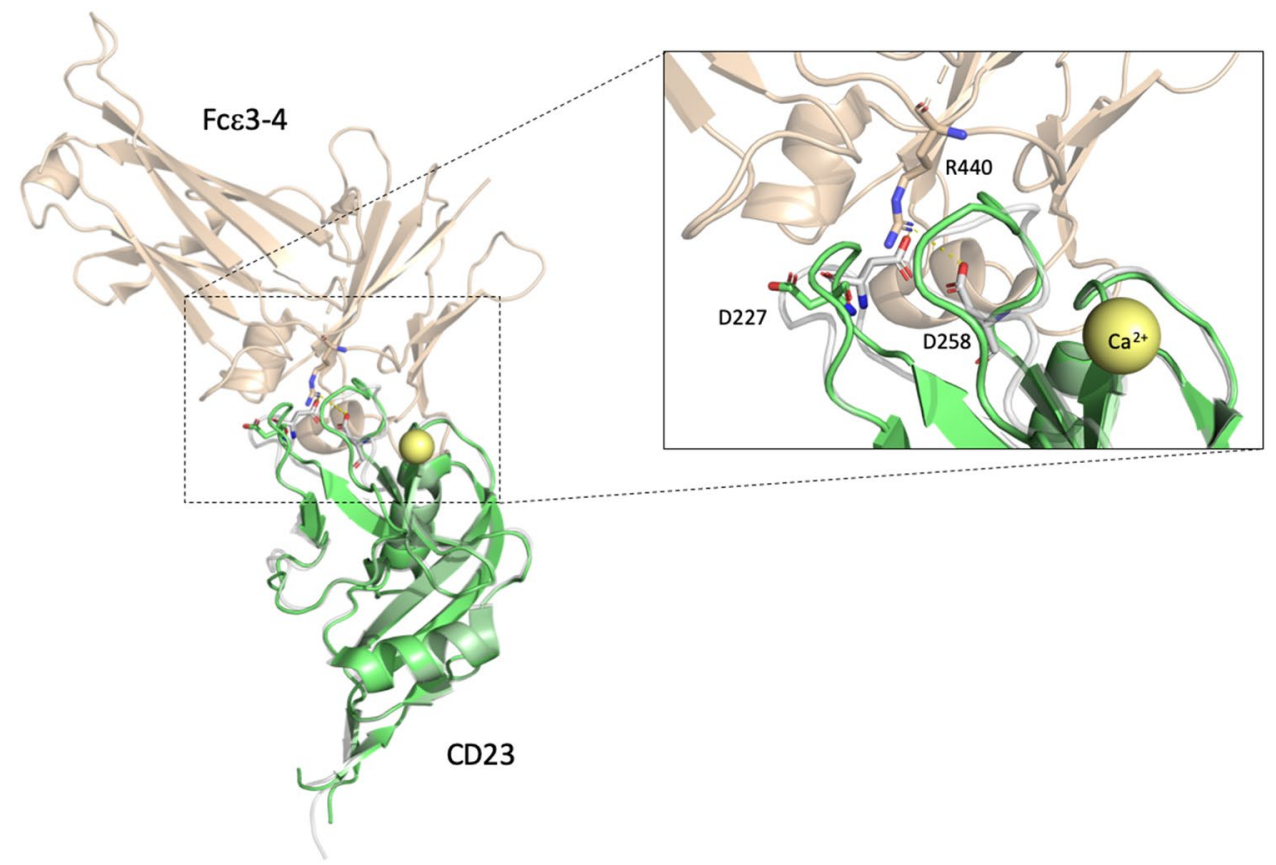

Figure 4. The superimposed structure containing CD23 apo and holo forms is obtained from the CD23 complex binding with $\mathrm{Ca}^{2+}$ and $\mathrm{Fc} \varepsilon 3-4$. The green cartoon represents the structure of the $\mathrm{Ca}^{2+}$-free wild-type CD23 lectin domain (PDB ID: 4G96) ${ }^{83}$. The structure of the CD23 holo form bound to $\mathrm{Ca}^{2+}$ complexed with Fce 3-4 (PDB ID: 4GKO) ${ }^{83}$ is drawn in gray- and wheat-colored cartoons. $\mathrm{Ca}^{2+}$ is shown in a yellow bubble; the magnified view shows the interface of CD23 and FcE 3-4. The D227 of the CD23 apo form is shown in the green stick. The salt bridges forming residues in the CD23 holo form and Fce 3-4 complex are highlighted with sticks.

GLU- and ASP-rich, as well as a $\mathrm{Ca}^{2+}$ buffering protein (Fig. 7a,b). Furthermore, higher WCN values were found in the microenvironment around E194 than in the third quartile of cancer-related SAVs in H-polar (RKEDQN), V-medium (NVEQIL), Z-low polarizability (GASDT), P-high polarity (HQRKNED), F-acidic (DE), and E-basic (HKR) descriptors and were lower than WCN values in the first quartile in E-sulfur-containing (CM) amino acid. Figure 7 depicts the microenvironment descriptor boxplots in the subgroup in which GLU mutates to GLY.

\section{Discussion}

Many cancer-related tools are based on genetic or protein sequence information, because of limited protein structure information. Next-generation sequencing technology has led to the large-scale application of genomic information in cancer research and human health ${ }^{90-93}$. The crucial limitation of this information is that while the technology may determine tumor risk or recurrence, cause and effect remain undetermined. The launch of the Human Proteome Project (HPP) has enriched our understanding of the human proteome blueprint responsible for complex diseases, including cancer. Approximately 20,000 human cancer-related proteome studies have been recorded in PubMed since $2011^{94}$. However, it remains very difficult to construct a 3-dimensional protein structure from these proteins, which explains why the widely used cancer prediction tools incorporate sequences of genetic information or sequence information of proteins. Importantly, the spatial conformation of protein forms the functional unit.

In this study, we describe how we developed a protein structure-based system, CanSavPre, to predict cancerrelated single amino acid variations. Protein sequence and structure descriptors are used in the model training process. Our prediction system displays excellent performance, and its structural and microenvironmental properties enable us to observe mutating amino acids that generate malfunctioning proteins. Critical descriptors emerge through use of the feature selection procedure. Figure 8 shows the heatmap of selected features in each training group of two prediction systems. For each descriptor, the color represents how many times were selected during four training repeats with different fitness functions. Mutated residues and their functional impact can be characterized by analyzing the selected feature sets. Although further study is needed to reveal the cancer mechanism in most selected features, our results indicate that it is possible to reliably predict cancer-related SAVs.

We also found that it is essential to divide the training data into proper subsets, according to the wild-type and mutated SAVs. Each amino acid with its unique characteristics plays a different role in protein construction. The heatmap in Fig. 8 also indicates that the profiles of selected features in CanSavPre $\mathrm{wm}_{\mathrm{wm}}$ are more legible than those in CanSavPre ${ }_{\mathrm{w}}$. The resulting discrepancies influence changes in protein conformation activity, to different degrees. The data from our two-level prediction system optimize the outcomes from a system that uses only 

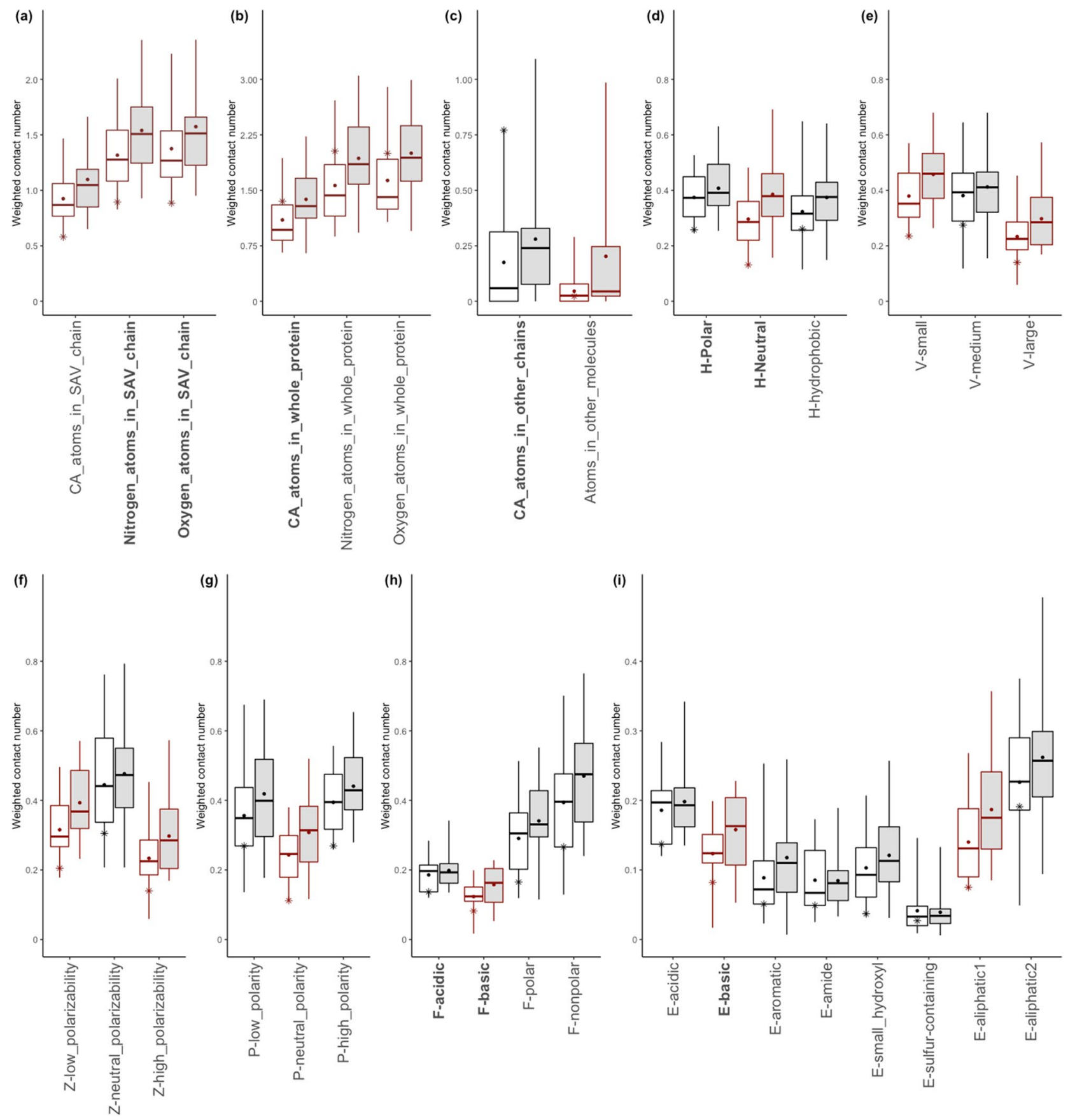

Figure 5. Boxplots of the microenvironment descriptors in the ASP that are altered to the TYR subgroup. All microenvironment descriptors are divided into nine groups: (a) atoms in the SAV chain; (b) atoms in whole protein; (c) atoms in other chains or molecules; (d) H-group; (e) V-group; (f) Z-group; (g) P-group; (h) F-group; and (i) E-group. The white and grey boxes represent the distribution of cancer-related and neutral SAVs. Red-framed boxes indicate that two sample $z$-testing revealed a significant difference between cancerrelated and neutral SAVs, with a 95\% confidence interval ( $\mathrm{z}$ score $>1.96, \mathrm{p}<0.05)$. Labels of descriptors selected by the genetic algorithm are bolded in the $x$-axis. Star symbols denote the D227Y cases in CD23.

one level. However, the complexities of protein function in the extensive cellular networks necessitate critical information that identifies cancer-related SAVs.

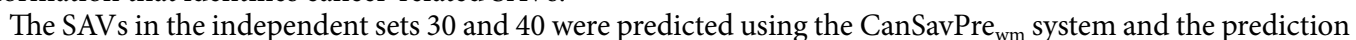
performance is illustrated in Table 8 . Although a difference in prediction performance is evident between the independent sets and the training set, this is largely because the performance is optimized by a genetic algorithm in the training set. Our purpose was to extract significant features using an optimization procedure. Thus, performance in the training set is the upper bound of prediction. Another reason for the difference in prediction performance between sets might be too few cancer-related SAVs in the training and independent sets. In the

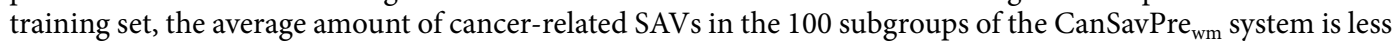




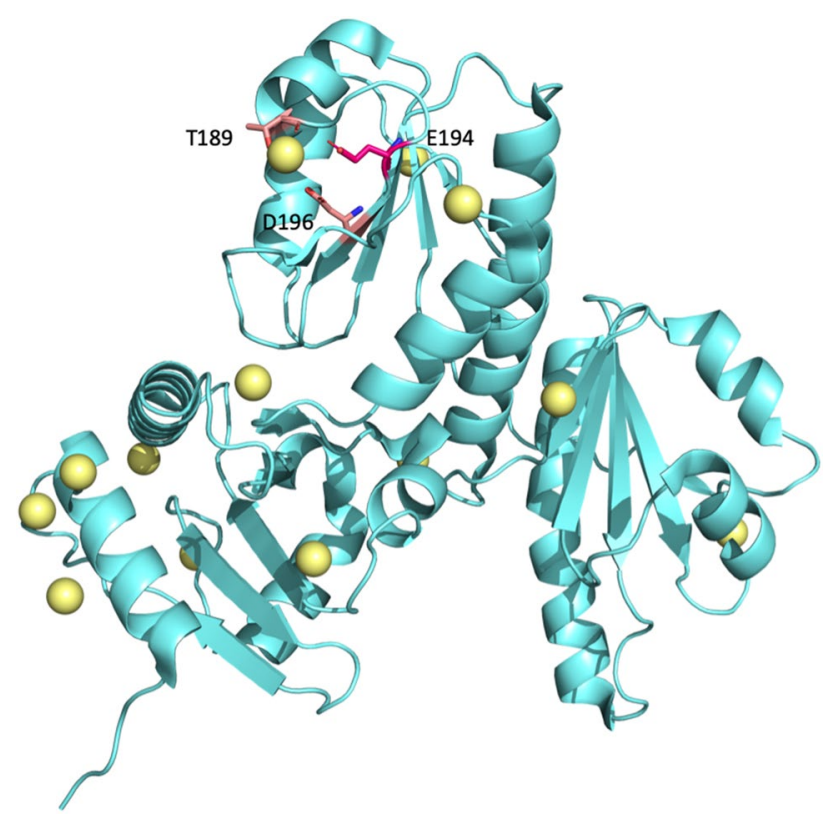

Figure 6. Protein structure of the human skeletal calsequestrin. The structure of CASQ (PDB ID: 3 UOM) ${ }^{89}$ in the cyan-colored cartoon is drawn by PyMOL. All of the yellow bubbles are $\mathrm{Ca}^{2+}$ in CASQ. Three $\mathrm{Ca}^{2+}$ binding residues are highlighted with sticks in deep pink. E194G is a cancer-related SAV.

than 30; the average amount is much smaller in the independent sets. The performance of independent sets might therefore be distorted, necessitating a greater number of cancer-related SAVs. Nevertheless, compared with DEOGEN2, which is mainly developed for deleterious amino acid variant prediction and publicly available, our prediction system performs better than DEOGEN2 in both two independent sets (Table 8).

Proteins are dynamic molecules with distinct, flexible structures that facilitate allosteric interactions between small molecules or proteins. For our prediction system, we focused on these interactions, i.e., physical contacts between proteins and molecules. A limitation of the crystal-protein structure is that it is capable of revealing only one condition of the protein-protein interaction. The critical purpose of a protein complex is to reflect interactions between single chain proteins. These interactions typically represent functional properties and are expected to be maintained through the genetic algorithm for feature selection. Importantly, technical limitations of the crystal structure prevent the formation of a protein complex in some conditions. Thus, some protein structures only include a part of the complex. These single chain proteins might restrict the range of our prediction system, because their conformations may differ from those of the protein complex. Our research seeks to define the phenomenon underlying cancer-related variations and how differences in conformations influence protein interactions, despite the limited available data on the protein complex.

Our case studies have used several different proteins to illustrate our protein structure-based system. In the first example, the PI3K protein family is well recognized for its association with cancer. The strict rule for our data extraction means that CD-HIT, the cluster database, filters out the homologous proteins and avoids weighting the mutation residues for the training model. Thus, the isoform PI3 $\mathrm{Kc} \boldsymbol{\delta}$ is selected as the representative isoform, even though PI3Kca is one of the most readily recognized isoforms in cancer research ${ }^{95,96}$. The most frequent and pathogenic mutation residues recorded in the TCGA, E542K and E545K, are predicted correctly in our system (see Supplementary Fig. S1). The evidence suggests that these two hotspot mutations induce glycolysis in cervical cancer cells via the $\beta$-catenin/SIRT3 signaling pathway ${ }^{97}$. Both glutamic acid mutations (E542K and E545K) are located in the helical domain. Biochemical studies have demonstrated that these mutated residues interact with p85a, so the alteration may affect the inhibitory activity of $\mathrm{p} 85 \mathrm{a}^{98}$.

Our prediction system also provides observations of the microenvironment for the SAV residues. The other case studies discussed in our results illustrate how the feature processes cope with the descriptors such as, for example, how the feature selection process manages ASP alteration patterns. This process also yields critical detail regarding ASP alterations (Fig. 4). ASP is a polar amino acid with a negative charge and the carboxyl group in the side chain of aspartic acid allows it to accept hydrogen atoms. Our model calculates the area surrounding the ASP. When the area surrounding the cancer-related ASP is unoccupied space, our model observes fewer basic amino acids compared with neutral ASP environments. This implies that, in this situation, the ASP might assume an interaction role with protein, whereby an ASP mutation can lead to an abnormal protein and different binding activity. This might explain how cellular changes result in tumor activity, as is illustrated in our second case study. In contrast, GLU is an amino acid with high polar residues and a negative charge that is also involved in the hydrogen atom acceptor role, with a distinctly different pattern to that of ASP (Fig. 6). Cancer-related GLUs are located in a denser region within a hydrophobic environment. Although ASP and GLU share similar characteristics, their different microenvironments justify construction of the prediction modules. 

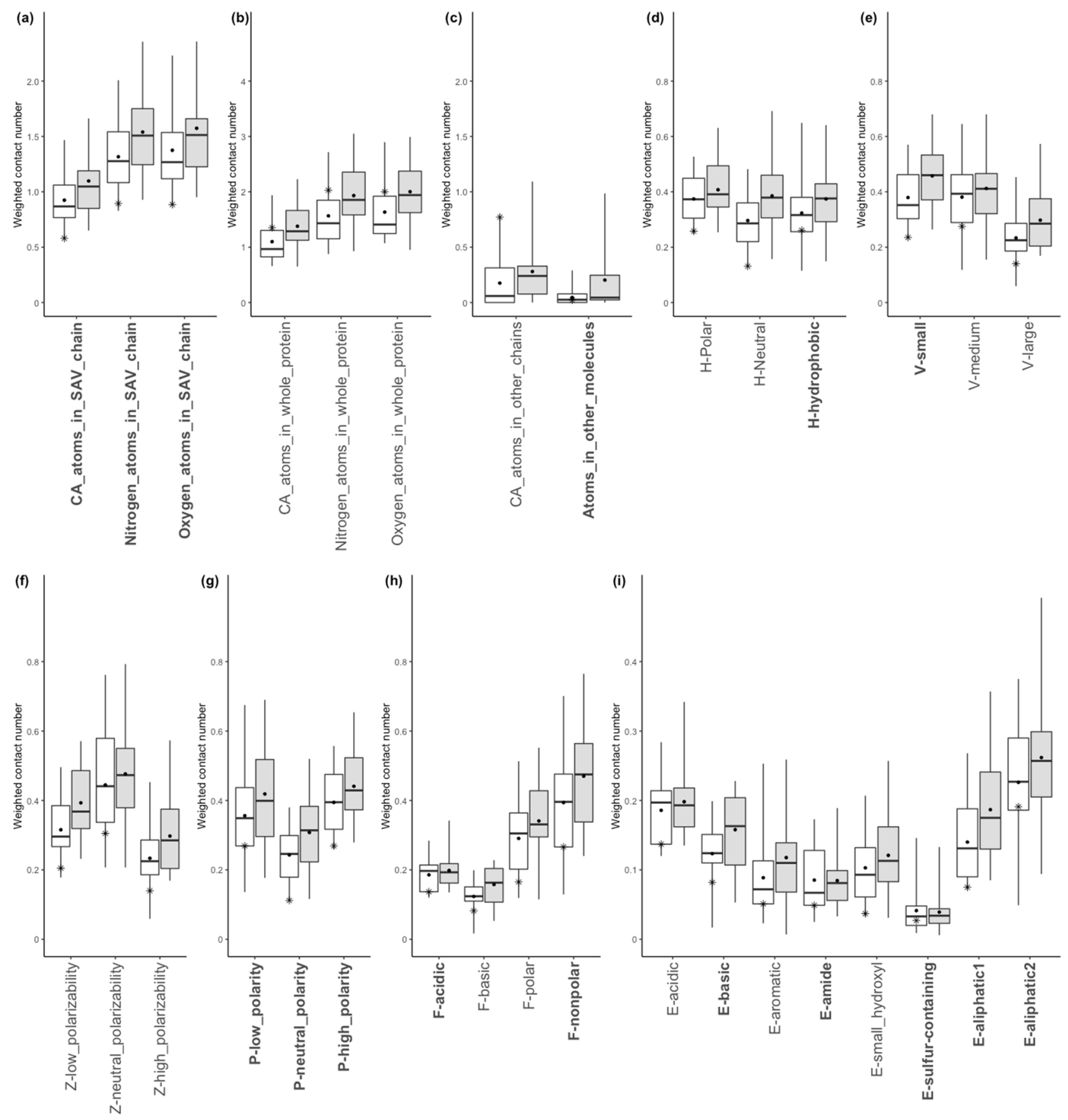

Figure 7. Boxplots of the microenvironment descriptors in the GLU that are altered to the GLY subgroup. All microenvironment descriptors are divided into nine groups: (a) atoms in SAV chain, (b) atoms in whole protein, (c) atoms in other chains or molecules, (d) H-group, (e) V-group, (f) Z-group, (g) P-group, (h) F-groups, and (i) E-groups. The white and grey boxes represented the distribution of cancer-related and neutral SAVs. The label of selected descriptors by the genetic algorithm are bold in the $x$-axis. The symbol stars denote the values of each feature in the case of E194G located at CASQ.

We suggest that protein structure and microenvironment features are the cornerstones of cancer research. Slight alterations in protein conformation properties may change the functional activity of molecular interactions. Clarifying protein structure is critical to understanding how a protein mutation can lead to cancer. However, the critical necessity for the protein structures is also the limitation of our prediction systems. Many emerging computational methods are attempting to clarify protein structure. For those proteins that only have sequence information, scientists can use a homology modeling method or an artificial intelligence system to extract the details needed for structural information. Notably, AlphaFold2 is considered to be an excellent solution to the problematic issue of protein folding ${ }^{99}$. As more protein structures become available over time, our model will benefit from the enriched databases, regardless of whether the data are sourced from experimental or predicted methods. 


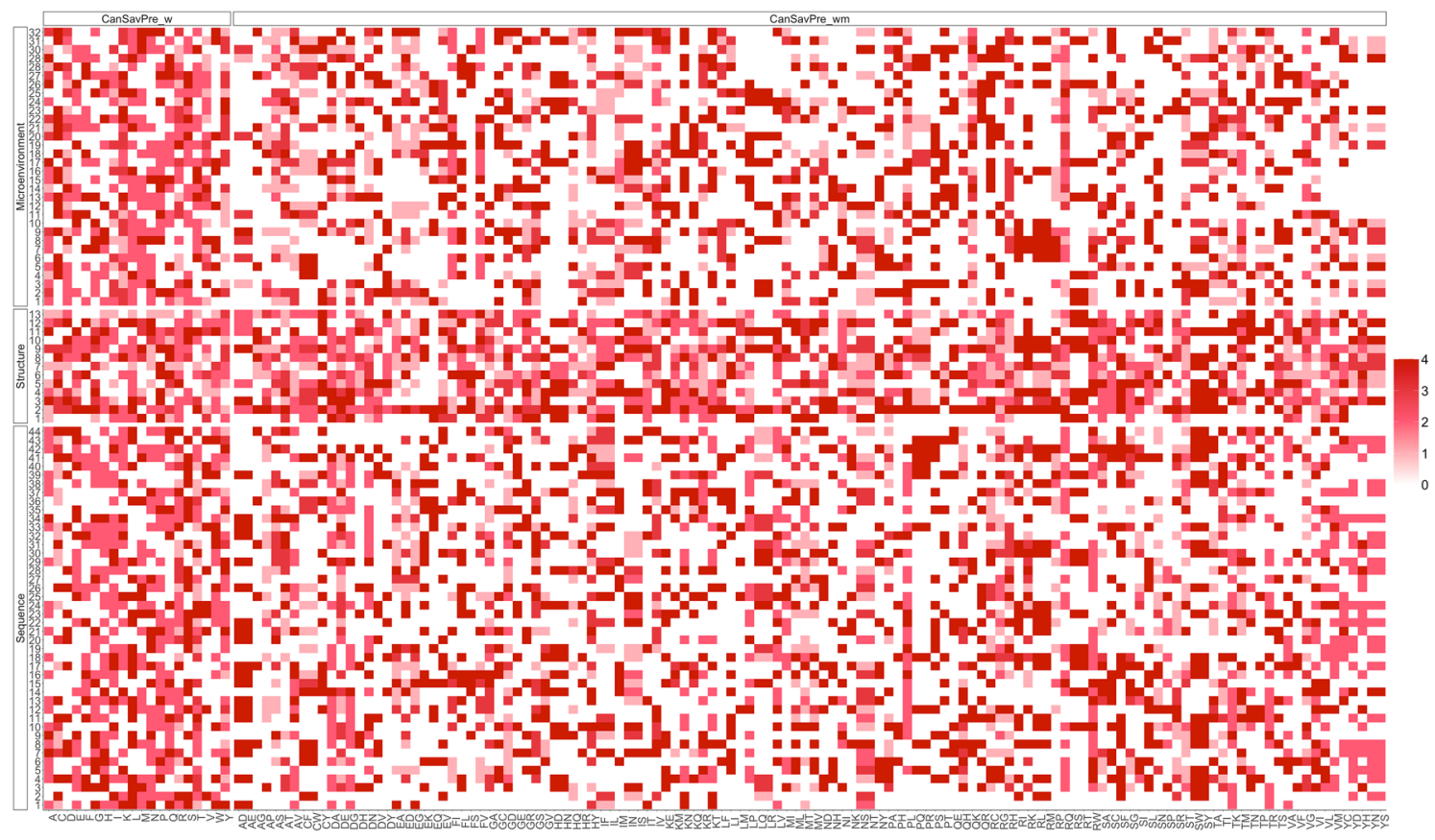

Figure 8. The heatmap of selected features in each training group of two prediction systems.

\begin{tabular}{|l|l|l|l|l|l|l|l|}
\hline Method & Dataset & Accuracy & Sensitivity & Specificity & MCC & Precision & F1 score \\
\hline CanSavPre & Independent set 30 & 0.8743 & 0.5844 & 0.8942 & 0.3419 & 0.2752 & 0.3742 \\
\hline CanSavPre & Independent set 40 & 0.8550 & 0.5839 & 0.8830 & 0.3699 & 0.3394 & 0.4292 \\
\hline DEOGEN2 & Independent set 30 & 0.7222 & 0.5517 & 0.7337 & 0.1539 & 0.1225 & 0.2005 \\
\hline DEOGEN2 & Independent set 40 & 0.6927 & 0.4693 & 0.7156 & 0.1170 & 0.1447 & 0.2212 \\
\hline
\end{tabular}

Table 8. Comparison of prediction performance values with DEOGEN2 in the independent sets.

In conclusion, we have developed a structure-based cancer-related single amino acid variation prediction system. This system not only displays excellent performance, but it also observes how the amino acid substitution influences protein activities. The descriptors provided by our system may offer targets for further research. Moreover, performance is markedly enhanced by the fact that the model includes conformation properties and details of the microenvironments surrounding SAVs. Furthermore, our algorithm detects the best combination of feature vectors for examining specific amino acid variations. Importantly, our model is a user-friendly web-based tool that scientists will find extremely useful when performing cancer research and precision medicine, particularly when investigating rare tumor mutations. The many genetic mutations in human cancers offer us numerous targets and possibilities that may be incorporated into our model, emphasizing its importance in cancer research.

Received: 18 March 2021; Accepted: 16 June 2021

Published online: 30 June 2021

\section{References}

1. Sunyaev, S., Ramensky, V. \& Bork, P. Towards a structural basis of human non-synonymous single nucleotide polymorphisms. Trends Genet. 16, 198-200 (2000).

2. Yue, P., Li, Z. \& Moult, J. Loss of protein structure stability as a major causative factor in monogenic disease. J. Mol. Biol. 353, 459-473. https://doi.org/10.1016/j.jmb.2005.08.020 (2005).

3. Juritz, E. et al. On the effect of protein conformation diversity in discriminating among neutral and disease related single amino acid substitutions. BMC Genom. 13(Suppl 4), S5. https://doi.org/10.1186/1471-2164-13-S4-S5 (2012).

4. Stitziel, N. O. et al. Structural location of disease-associated single-nucleotide polymorphisms. J. Mol. Biol. 327, 1021-1030. https://doi.org/10.1016/s0022-2836(03)00240-7 (2003).

5. Teng, S., Srivastava, A. K., Schwartz, C. E., Alexov, E. \& Wang, L. Structural assessment of the effects of amino acid substitutions on protein stability and protein protein interaction. Int. J. Comput. Biol. Drug Des. 3, 334-349. https://doi.org/10.1504/IJCBDD. 2010.038396 (2010).

6. Redler, R. L., Das, J., Diaz, J. R. \& Dokholyan, N. V. Protein destabilization as a common factor in diverse inherited disorders. J. Mol. Evol. 82, 11-16. https://doi.org/10.1007/s00239-015-9717-5 (2016).

7. Ponzoni, L. \& Bahar, I. Structural dynamics is a determinant of the functional significance of missense variants. Proc. Natl. Acad. Sci. U S A 115, 4164-4169. https://doi.org/10.1073/pnas.1715896115 (2018). 
8. Bromberg, Y. \& Rost, B. Correlating protein function and stability through the analysis of single amino acid substitutions. BMC Bioinform. 10, S8. https://doi.org/10.1186/1471-2105-10-S8-S8 (2009).

9. David, A., Razali, R., Wass, M. N. \& Sternberg, M. J. Protein-protein interaction sites are hot spots for disease-associated nonsynonymous SNPs. Hum. Mutat. 33, 359-363. https://doi.org/10.1002/humu.21656 (2012).

10. Yates, C. M. \& Sternberg, M. J. Proteins and domains vary in their tolerance of non-synonymous single nucleotide polymorphisms (nsSNPs). J. Mol. Biol. 425, 1274-1286. https://doi.org/10.1016/j.jmb.2013.01.026 (2013).

11. Niroula, A. \& Vihinen, M. Harmful somatic amino acid substitutions affect key pathways in cancers. BMC Med Genom. 8, 53. https://doi.org/10.1186/s12920-015-0125-x (2015).

12. Lori, C. et al. Effect of single amino acid substitution observed in cancer on Pim-1 kinase thermodynamic stability and structure. PLoS ONE 8, e64824. https://doi.org/10.1371/journal.pone.0064824 (2013).

13. Song, C. et al. Large-scale quantification of single amino-acid variations by a variation-associated database search strategy. J. Proteome Res. 13, 241-248. https://doi.org/10.1021/pr400544j (2014).

14. Cancer Genome Atlas Research, N. Integrated genomic analyses of ovarian carcinoma. Nature 474, 609-615. https://doi.org/ 10.1038/nature10166 (2011).

15. Cancer Genome Atlas Research, N. Comprehensive molecular profiling of lung adenocarcinoma. Nature 511, 543-550. https:// doi.org/10.1038/nature13385 (2014).

16. Balmain, A. The critical roles of somatic mutations and environmental tumor-promoting agents in cancer risk. Nat. Genet. 52, 1139-1143. https://doi.org/10.1038/s41588-020-00727-5 (2020).

17. McFarland, C. D. et al. The damaging effect of passenger mutations on cancer progression. Cancer Res. 77, 4763-4772. https:// doi.org/10.1158/0008-5472.CAN-15-3283-T (2017).

18. Chen, J. et al. Genetic regulatory subnetworks and key regulating genes in rat hippocampus perturbed by prenatal malnutrition: Implications for major brain disorders. Aging (Albany NY) 12, 8434-8458. https://doi.org/10.18632/aging.103150 (2020).

19. Li, H. et al. Co-expression network analysis identified hub genes critical to triglyceride and free fatty acid metabolism as key regulators of age-related vascular dysfunction in mice. Aging (Albany NY) 11, 7620-7638. https://doi.org/10.18632/aging.102275 (2019).

20. Son, H., Kang, H., Kim, H. S. \& Kim, S. Somatic mutation driven codon transition bias in human cancer. Sci. Rep. 7, 14204. https://doi.org/10.1038/s41598-017-14543-1 (2017).

21. Tsuber, V., Kadamov, Y., Brautigam, L., Berglund, U. W. \& Helleday, T. Mutations in cancer cause gain of cysteine, histidine, and tryptophan at the expense of a net loss of arginine on the proteome level. Biomolecules https://doi.org/10.3390/biom7030049 (2017).

22. Anoosha, P., Sakthivel, R. \& Michael Gromiha, M. Exploring preferred amino acid mutations in cancer genes: Applications to identify potential drug targets. Biochim. Biophys. Acta 1862, 155-165. https://doi.org/10.1016/j.bbadis.2015.11.006 (2016).

23. Halasi, M. et al. ROS inhibitor N-acetyl-L-cysteine antagonizes the activity of proteasome inhibitors. Biochem. J. 454, 201-208. https://doi.org/10.1042/BJ20130282 (2013).

24. Szpiech, Z. A. et al. Prominent features of the amino acid mutation landscape in cancer. PLoS ONE 12, e0183273. https://doi. org/10.1371/journal.pone.0183273 (2017).

25. Tan, H., Bao, J. \& Zhou, X. Genome-wide mutational spectra analysis reveals significant cancer-specific heterogeneity. Sci. Rep. 5, 12566. https://doi.org/10.1038/srep12566 (2015).

26. Yu, H. et al. LEPR hypomethylation is significantly associated with gastric cancer in males. Exp. Mol. Pathol. 116, 104493. https:// doi.org/10.1016/j.yexmp.2020.104493 (2020).

27. Liu, M. et al. A multi-model deep convolutional neural network for automatic hippocampus segmentation and classification in Alzheimer's disease. Neuroimage 208, 116459. https://doi.org/10.1016/j.neuroimage.2019.116459 (2020).

28. Mularoni, L., Sabarinathan, R., Deu-Pons, J., Gonzalez-Perez, A. \& Lopez-Bigas, N. OncodriveFML: A general framework to identify coding and non-coding regions with cancer driver mutations. Genome Biol. 17, 128. https://doi.org/10.1186/s13059016-0994-0 (2016).

29. Wang, Z. et al. Cancer driver mutation prediction through Bayesian integration of multi-omic data. PLoS ONE 13, e0196939. https://doi.org/10.1371/journal.pone.0196939 (2018).

30. Richards, S. et al. Standards and guidelines for the interpretation of sequence variants: A joint consensus recommendation of the American College of Medical Genetics and Genomics and the Association for Molecular Pathology. Genet. Med. 17, 405-424. https://doi.org/10.1038/gim.2015.30 (2015).

31. Qian, D. et al. A Bayesian framework for efficient and accurate variant prediction. PLoS ONE 13, e0203553. https://doi.org/10. 1371/journal.pone.0203553 (2018).

32. Chen, H. et al. Comprehensive assessment of computational algorithms in predicting cancer driver mutations. Genome Biol. 21, 43. https://doi.org/10.1186/s13059-020-01954-z (2020).

33. Carter, H. et al. Cancer-specific high-throughput annotation of somatic mutations: Computational prediction of driver missense mutations. Cancer Res. 69, 6660-6667. https://doi.org/10.1158/0008-5472.CAN-09-1133 (2009).

34. Raimondi, D. et al. DEOGEN2: Prediction and interactive visualization of single amino acid variant deleteriousness in human proteins. Nucleic Acids Res. 45, W201-W206. https://doi.org/10.1093/nar/gkx390 (2017).

35. Bailey, M. H. et al. Comprehensive characterization of cancer driver genes and mutations. Cell 174, 1034-1035. https://doi.org/ 10.1016/j.cell.2018.07.034 (2018)

36. Care, M. A., Needham, C. J., Bulpitt, A. J. \& Westhead, D. R. Deleterious SNP prediction: Be mindful of your training data!. Bioinformatics 23, 664-672. https://doi.org/10.1093/bioinformatics/btl649 (2007).

37. Li, J. et al. A bioinformatics workflow for variant peptide detection in shotgun proteomics. Mol. Cell Proteom. 10, M110006536. https://doi.org/10.1074/mcp.M110.006536 (2011).

38. Zhang, M. et al. CanProVar 2.0: An updated database of human cancer proteome variation. J. Proteome Res. 16, 421-432. https:// doi.org/10.1021/acs.jproteome.6b00505 (2017).

39. O’Donovan, C., Apweiler, R. \& Bairoch, A. The human proteomics initiative (HPI). Trends Biotechnol. 19, 178-181. https://doi. org/10.1016/s0167-7799(01)01598-0 (2001).

40. Bamford, S. et al. The COSMIC (Catalogue of Somatic Mutations in Cancer) database and website. Br. J. Cancer 91, 355-358. https://doi.org/10.1038/sj.bjc.6601894 (2004).

41. Hamosh, A., Scott, A. F., Amberger, J. S., Bocchini, C. A. \& McKusick, V. A. Online Mendelian Inheritance in Man (OMIM), a knowledgebase of human genes and genetic disorders. Nucleic Acids Res. 33, D514-517. https://doi.org/10.1093/nar/gki033 (2005).

42. Cancer Genome Atlas Research, N. Comprehensive genomic characterization defines human glioblastoma genes and core pathways. Nature 455, 1061-1068. https://doi.org/10.1038/nature07385 (2008).

43. Greenman, C. et al. Patterns of somatic mutation in human cancer genomes. Nature 446, 153-158. https://doi.org/10.1038/natur e05610 (2007).

44. Sjoblom, T. et al. The consensus coding sequences of human breast and colorectal cancers. Science 314, 268-274. https://doi. org/10.1126/science.1133427 (2006).

45. Altschul, S. F., Gish, W., Miller, W., Myers, E. W. \& Lipman, D. J. Basic local alignment search tool. J. Mol. Biol. 215, 403-410. https://doi.org/10.1016/S0022-2836(05)80360-2 (1990). 
46. Huang, Y., Niu, B., Gao, Y., Fu, L. \& Li, W. CD-HIT Suite: A web server for clustering and comparing biological sequences. Bioinformatics 26, 680-682. https://doi.org/10.1093/bioinformatics/btq003 (2010).

47. Hua, S. \& Sun, Z. A novel method of protein secondary structure prediction with high segment overlap measure: Support vector machine approach. J. Mol. Biol. 308, 397-407 (2001).

48. Yu, C. S. et al. Fine-grained protein fold assignment by support vector machines using generalized npeptide coding schemes and jury voting from multiple-parameter sets. Proteins 50, 531-536. https://doi.org/10.1002/prot.10313 (2003).

49. Yu, C. S., Lin, C. J. \& Hwang, J. K. Predicting subcellular localization of proteins for Gram-negative bacteria by support vector machines based on n-peptide compositions. Protein Sci. 13, 1402-1406. https://doi.org/10.1110/ps.03479604 (2004).

50. Chen, Y. C., Lin, Y. S., Lin, C. J. \& Hwang, J. K. Prediction of the bonding states of cysteines using the support vector machines based on multiple feature vectors and cysteine state sequences. Proteins 55, 1036-1042. https://doi.org/10.1002/prot.20079 (2004).

51. Lei, Z. \& Dai, Y. An SVM-based system for predicting protein subnuclear localizations. BMC Bioinform. 6, 291. https://doi.org/ 10.1186/1471-2105-6-291 (2005)

52. Ward, J. J., McGuffin, L. J., Buxton, B. F. \& Jones, D. T. Secondary structure prediction with support vector machines. Bioinformatics 19, 1650-1655 (2003).

53. Chang, C. C. \& Lin, C. J. LIBSVM: A library for support vector machines. Acm Trans. Intell. Syst. Technol. 2, 27. https://doi.org/ 10.1145/1961189.1961199 (2011).

54. Lin, C.-J. Formulations of support vector machines: A note from an optimization point of view. Neural Comput. 13, 307-317 (2001).

55. Lu, C. H., Chen, Y. C., Yu, C. S. \& Hwang, J. K. Predicting disulfide connectivity patterns. Proteins 67, 262-270. https://doi.org/ 10.1002/prot.21309 (2007).

56. Yu, C. S. \& Lu, C. H. Identification of antifreeze proteins and their functional residues by support vector machine and genetic algorithms based on n-peptide compositions. PLoS ONE 6, e20445. https://doi.org/10.1371/journal.pone.0020445 (2011).

57. Choi, Y., Sims, G. E., Murphy, S., Miller, J. R. \& Chan, A. P. Predicting the functional effect of amino acid substitutions and indels. PLOS ONE 7, e46688. https://doi.org/10.1371/journal.pone.0046688 (2012).

58. Henikoff, S. \& Henikoff, J. G. Amino acid substitution matrices from protein blocks. Proc. Natl. Acad. Sci. U S A 89, 10915-10919 (1992).

59. Jones, D. T., Taylor, W. R. \& Thornton, J. M. The rapid generation of mutation data matrices from protein sequences. Comput. Appl. Biosci. 8, 275-282 (1992).

60. Altschul, S. F. et al. Gapped BLAST and PSI-BLAST: A new generation of protein database search programs. Nucleic Acids Res. 25, 3389-3402 (1997)

61. Chou, K. C. Prediction of protein cellular attributes using pseudo-amino acid composition. Proteins 43, 246-255 (2001).

62. Yu, C. S., Chen, Y. C., Lu, C. H. \& Hwang, J. K. Prediction of protein subcellular localization. Proteins 64, 643-651. https://doi. org/10.1002/prot.21018 (2006).

63. Cheng, J., Randall, A. Z., Sweredoski, M. J. \& Baldi, P. SCRATCH: A protein structure and structural feature prediction server. Nucleic Acids Res. 33, W72-76. https://doi.org/10.1093/nar/gki396 (2005).

64. Kabsch, W. \& Sander, C. Dictionary of protein secondary structure: Pattern recognition of hydrogen-bonded and geometrical features. Biopolymers 22, 2577-2637. https://doi.org/10.1002/bip.360221211 (1983).

65. Lin, C. P. et al. Deriving protein dynamical properties from weighted protein contact number. Proteins 72, 929-935. https://doi. org/10.1002/prot.21983 (2008).

66. Shih, C. H., Chang, C. M., Lin, Y. S., Lo, W. C. \& Hwang, J. K. Evolutionary information hidden in a single protein structure. Proteins 80, 1647-1657. https://doi.org/10.1002/prot.24058 (2012).

67. Hemmings, B. A. \& Restuccia, D. F. PI3K-PKB/Akt pathway. Cold Spring Harb. Perspect. Biol. 4, a011189. https://doi.org/10. 1101/cshperspect.a011189 (2012).

68. Dong, P. et al. The impact of microRNA-mediated PI3K/AKT signaling on epithelial-mesenchymal transition and cancer stemness in endometrial cancer. J. Transl. Med. 12, 231. https://doi.org/10.1186/s12967-014-0231-0 (2014).

69. Asati, V., Mahapatra, D. K. \& Bharti, S. K. PI3K/Akt/mTOR and Ras/Raf/MEK/ERK signaling pathways inhibitors as anticancer agents: Structural and pharmacological perspectives. Eur. J. Med. Chem. 109, 314-341. https://doi.org/10.1016/j.ejmech.2016. 01.012 (2016).

70. Benetatos, L., Voulgaris, E. \& Vartholomatos, G. The crosstalk between long non-coding RNAs and PI3K in cancer. Med. Oncol. 34, 39. https://doi.org/10.1007/s12032-017-0897-2 (2017).

71. Dong, T. et al. The expression of CD9 and PIK3CD is associated with prognosis of follicular lymphoma. J. Cancer 6, 1222-1229. https://doi.org/10.7150/jca.11279 (2015)

72. Chen, J. S. et al. PIK3CD induces cell growth and invasion by activating AKT/GSK-3beta/beta-catenin signaling in colorectal cancer. Cancer Sci. 110, 997-1011. https://doi.org/10.1111/cas.13931 (2019).

73. Heffron, T. P. et al. The rational design of selective benzoxazepin inhibitors of the alpha-isoform of phosphoinositide 3-kinase culminating in the identification of (S)-2-((2-(1-Isopropyl-1H-1,2,4-triazol-5-yl)-5,6-dihydrobenzo[f]imidazo[1,2-d][1,4] oxazepin-9-yl)oxy)propanamide (GDC-0326). J. Med. Chem. 59, 985-1002. https://doi.org/10.1021/acs.jmedchem.5b01483 (2016).

74. Acharya, M. et al. CD23/FcepsilonRII: Molecular multi-tasking. Clin. Exp. Immunol. 162, 12-23. https://doi.org/10.1111/j. 1365-2249.2010.04210.x (2010).

75. Delespesse, G. et al. Expression, structure, and function of the CD23 antigen. Adv. Immunol. 49, 149-191. https://doi.org/10. 1016/s0065-2776(08)60776-2 (1991).

76. Vercelli, D. et al. Human recombinant interleukin 4 induces Fc epsilon R2/CD23 on normal human monocytes. J. Exp. Med. 167, 1406-1416. https://doi.org/10.1084/jem.167.4.1406 (1988)

77. Krauss, S., Mayer, E., Rank, G. \& Rieber, E. P. Induction of the low affinity receptor for IgE (Fc epsilon RII/CD23) on human blood dendritic cells by interleukin-4. Adv. Exp. Med. Biol. 329, 231-236. https://doi.org/10.1007/978-1-4615-2930-9 39 (1993).

78. Rieber, E. P., Rank, G., Kohler, I. \& Krauss, S. Membrane expression of Fc epsilon RII/CD23 and release of soluble CD23 by follicular dendritic cells. Adv. Exp. Med. Biol. 329, 393-398 (1993).

79. Fourcade, C. et al. Expression of CD23 by human bone marrow stromal cells. Eur. Cytokine Netw. 3, 539-543 (1992).

80. Stransky, N. et al. The mutational landscape of head and neck squamous cell carcinoma. Science 333, 1157-1160. https://doi. org/10.1126/science.1208130 (2011)

81. Woischke, C. et al. In-depth mutational analyses of colorectal neuroendocrine carcinomas with adenoma or adenocarcinoma components. Mod. Pathol. 30, 95-103. https://doi.org/10.1038/modpathol.2016.150 (2017).

82. Dhaliwal, B. et al. Conformational plasticity at the IgE-binding site of the B-cell receptor CD23. Mol. Immunol. 56, 693-697. https://doi.org/10.1016/j.molimm.2013.07.005 (2013).

83. Yuan, D. et al. $\mathrm{Ca} 2+$-dependent structural changes in the B-cell receptor $\mathrm{CD} 23$ increase its affinity for human immunoglobulin E. J. Biol. Chem. 288, 21667-21677. https://doi.org/10.1074/jbc.M113.480657 (2013).

84. MacLennan, D. H., Abu-Abed, M. \& Kang, C. Structure-function relationships in $\mathrm{Ca}(2+)$ cycling proteins. J. Mol. Cell. Cardiol. 34, 897-918. https://doi.org/10.1006/jmcc.2002.2031 (2002). 
85. Kim, E., Tam, M., Siems, W. F. \& Kang, C. Effects of drugs with muscle-related side effects and affinity for calsequestrin on the calcium regulatory function of sarcoplasmic reticulum microsomes. Mol. Pharmacol. 68, 1708-1715. https://doi.org/10.1124/ mol.105.016253 (2005).

86. Manno, C. et al. Calsequestrin depolymerizes when calcium is depleted in the sarcoplasmic reticulum of working muscle. Proc. Natl. Acad. Sci. U S A 114, E638-E647. https://doi.org/10.1073/pnas.1620265114 (2017)

87. Terentyev, D. et al. Calsequestrin determines the functional size and stability of cardiac intracellular calcium stores: Mechanism for hereditary arrhythmia. Proc. Natl. Acad. Sci. USA 100, 11759-11764. https://doi.org/10.1073/pnas.1932318100 (2003).

88. Stewart, T. A., Yapa, K. T. \& Monteith, G. R. Altered calcium signaling in cancer cells. Biochim. Biophys. Acta 8481, 2502-2511. https://doi.org/10.1016/j.bbamem.2014.08.016 (2015).

89. Sanchez, E. J., Lewis, K. M., Danna, B. R. \& Kang, C. High-capacity Ca2+ binding of human skeletal calsequestrin. J. Biol. Chem. 287, 11592-11601. https://doi.org/10.1074/jbc.M111.335075 (2012).

90. Wu, Y. et al. Multi-trait analysis for genome-wide association study of five psychiatric disorders. Transl. Psychiatry 10, 209. https://doi.org/10.1038/s41398-020-00902-6 (2020).

91. Zheng, S. et al. Immunodeficiency promotes adaptive alterations of host gut microbiome: An observational metagenomic study in mice. Front. Microbiol. 10, 2415. https://doi.org/10.3389/fmicb.2019.02415 (2019).

92. Zhang, F. et al. Causal influences of neuroticism on mental health and cardiovascular disease. Hum. Genet. https://doi.org/10. 1007/s00439-021-02288-x (2021).

93. Zhang, F. et al. Genetic evidence suggests posttraumatic stress disorder as a subtype of major depressive disorder. J. Clin. Invest. https://doi.org/10.1172/JCI145942 (2021).

94. Adhikari, S. et al. A high-stringency blueprint of the human proteome. Nat. Commun. 11, 5301. https://doi.org/10.1038/s41467020-19045-9 (2020).

95. Samuels, Y. \& Waldman, T. Oncogenic mutations of PIK3CA in human cancers. Curr. Top. Microbiol. Immunol. 347, 21-41. https://doi.org/10.1007/82_2010_68 (2010).

96. Samuels, Y. \& Ericson, K. Oncogenic PI3K and its role in cancer. Curr. Opin. Oncol. 18, 77-82. https://doi.org/10.1097/01.cco. 0000198021.99347.b9 (2006).

97. Jiang, W. et al. The PIK3CA E542K and E545K mutations promote glycolysis and proliferation via induction of the beta-catenin/ SIRT3 signaling pathway in cervical cancer. J. Hematol. Oncol. 11, 139. https://doi.org/10.1186/s13045-018-0674-5 (2018).

98. Ligresti, G. et al. PIK3CA mutations in human solid tumors: Role in sensitivity to various therapeutic approaches. Cell Cycle 8, 1352-1358. https://doi.org/10.4161/cc.8.9.8255 (2009).

99. Callaway, E. “It will change everything”: DeepMind's AI makes gigantic leap in solving protein structures. Nature 588, 203-204. https://doi.org/10.1038/d41586-020-03348-4 (2020).

100. Schrödinger, L. The PyMOL Molecular Graphics System, Version 1.8. (2015).

\section{Acknowledgements}

This work was supported by the Ministry of Science and Technology, Taipei, Taiwan (Grant No. MOST 108-2221E-039-013-) and by China Medical University, Taichung, Taiwan (Grant No. CMU-108-MF-121).

\section{Author contributions}

J.J. Liu and H.W. Wu performed the formal analysis and investigation. J.J. Liu and Y.J. Chang analyzed the data, prepared figures, and wrote the draft. J.J. Liu and C.S. Yu designed the website. C.H. Lu, C.S. Yu and C.P. Lin reviewed the manuscript. C.H. Lu supervised and designed this study.

\section{Funding}

Ministry of Science Technology, Taiwan, Grant Number: MOST 108-2221-E-039-013-; China Medical University, Taiwan, Grant Number: CMU-108-MF-121.

\section{Competing interests}

The authors declare no competing interests.

\section{Additional information}

Supplementary Information The online version contains supplementary material available at https://doi.org/ 10.1038/s41598-021-92793-w.

Correspondence and requests for materials should be addressed to C.-H.L.

Reprints and permissions information is available at www.nature.com/reprints.

Publisher's note Springer Nature remains neutral with regard to jurisdictional claims in published maps and institutional affiliations.

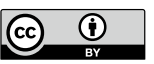

Open Access This article is licensed under a Creative Commons Attribution 4.0 International License, which permits use, sharing, adaptation, distribution and reproduction in any medium or format, as long as you give appropriate credit to the original author(s) and the source, provide a link to the Creative Commons licence, and indicate if changes were made. The images or other third party material in this article are included in the article's Creative Commons licence, unless indicated otherwise in a credit line to the material. If material is not included in the article's Creative Commons licence and your intended use is not permitted by statutory regulation or exceeds the permitted use, you will need to obtain permission directly from the copyright holder. To view a copy of this licence, visit http://creativecommons.org/licenses/by/4.0/.

(C) The Author(s) 2021 